\title{
Vector-valued operators with singular kernel and Triebel-Lizorkin block spaces with variable exponents
}

\author{
Kwok-Pun Ho
}

\begin{abstract}
We establish two principles which state that, whenever an operator is bounded on a given Banach function space, then under some simple conditions, it is also bounded on the corresponding Morrey spaces and block spaces. By applying these principles on some concrete operators, we generalize the Fefferman-Stein vector-valued inequalities, define and study the Triebel-Lizorkin block spaces with variable exponents, and extend the mapping properties of the fractional integral operators to Morrey-type spaces and block-type spaces.
\end{abstract}

\section{Introduction}

In this paper, we establish two main results on the boundedness of the vectorvalued operators on Morrey-type spaces and block-type spaces. Our results apply to some important operators such as the singular integral operators, the Calderón-Zygmund operators, and the fractional integral operators. Furthermore, we also have the boundedness result for the corresponding vector-valued operators. As an application of these boundedness results, we introduce and study the Triebel-Lizorkin block spaces with variable exponents.

Recently, there has been a substantial amount of research on generalizing the boundedness of some important operators on Lebesgue spaces to general function spaces. For instance, the results in [3], [9], [12], [13], [18], [28], [29], [35], [43], [51], and [55] give us the boundedness of some important operators on Lebesgue spaces, Morrey spaces, and block spaces in the variable exponent setting.

The classical Morrey space was introduced by Morrey [47] for the study of elliptic partial differential equations. Since then, the Morrey space has become one of the most important function spaces in analysis.

For the classical Morrey spaces, the boundedness of the vector-valued HardyLittlewood maximal operator, namely, the Fefferman-Stein vector-valued maximal inequalities, was established in [65] and [70]. By using these inequalities, the Triebel-Lizorkin-Morrey spaces were introduced in [65] and [70]. Note that there is another family of Triebel-Lizorkin-Morrey spaces (see [74]-[76]). The

Kyoto Journal of Mathematics, Vol. 56, No. 1 (2016), 97-124

DOI 10.1215/21562261-3445165, (C) 2016 by Kyoto University

Received September 29, 2014. Revised December 16, 2014. Accepted December 16, 2014.

2010 Mathematics Subject Classification: Primary 42B20, 42B25; Secondary 47B38, 47G10. 
Triebel-Lizorkin-Morrey spaces are referred to as Triebel-Lizorkin-type spaces in [67]. Even though it was shown in [67] that these two families are the same, the development of these two families had different motivations.

The Fefferman-Stein vector-valued maximal inequalities can be further extended to Morrey spaces with variable exponents; this result was presented in [39]. For the Morrey spaces with variable exponents, the boundedness of the Hardy-Littlewood maximal function was given in [3], [30], and [43].

In addition, the family of Triebel-Lizorkin-Morrey spaces was also generalized in [35] to the family of Triebel-Lizorkin-Morrey spaces with variable exponents. This extension was based on generalizing the boundedness results for some vector-valued singular integral operators introduced in [17].

Instead of the classical Morrey space, there is another natural extension of Lebesgue space, namely, the block space (see [7]). Block spaces also have a connection to the classical Morrey spaces. The dual spaces of block spaces are the classical Morrey spaces (see [7, Theorem 1], [42], [77]). The mapping properties of the fractional integral operators on the classical Morrey spaces and the classical block spaces were developed in [2] and [1].

The reader is reminded that, in [46] and [68], the term block space was used to represent another family of function spaces. The classical block space was generalized to the block spaces with variable exponents in [9]. Additionally, the boundedness of the Hardy-Littlewood maximal operator on the block spaces with variable exponents was obtained in [9].

We find that the above results and theorems for Morrey spaces and block spaces are consequences of or rely on the boundedness of some vector-valued operators on the corresponding Morrey spaces and block spaces. This motivates us to establish a general principle to obtain the boundedness of some operators on Morrey spaces and block spaces.

Roughly speaking, our main results in this paper find that, whenever an operator is bounded on a Banach function space $X$ (see Definition 2.1), then under some mild conditions, this operator is also bounded on the corresponding Morrey-type spaces $\mathcal{M}_{u}^{X}$ and the corresponding block-type spaces $\mathfrak{B}_{u, X}$. Most importantly, investigating such an abstract setting is not a mere quest to generalization, it also has potential applications on partial differential equations (for instance, the reader may consult [66] for the generalization of the GagliardoNirenberg inequality to the Sobolev-Morrey spaces).

The above idea of extending the boundedness of operators from a given Banach function space to the corresponding Morrey-type spaces is inspired by the boundedness of the Hardy-Littlewood maximal operator in the classical Morrey spaces (see [10]), the mapping properties for the singular integral operators on the classical Morrey spaces (see [48]), and the Spanne-type result for the fractional integral operators on Morrey spaces (see [57]).

Our main result on the boundedness of some vector-valued operators gives us several interesting applications. The Fefferman-Stein vector-valued maximal inequalities are extended to the Morrey spaces and block spaces associated with 
general Banach function space. This extension includes the results in [33], [65], and [70]. Next, we find that the results in [17] for the Triebel-Lizorkin spaces with variable exponents depend on the boundedness of some vector-valued operators. Applying our main results to these operators, we can define the Triebel-Lizorkin block spaces with variable exponents and study some of their important properties such as the boundedness of the $\phi-\psi$ transforms, the atomic decompositions, and the molecular characterizations. In fact, this method was already employed in [35] to introduce and study the Triebel-Lizorkin-Morrey spaces with variable exponents. Finally, our main theorems also apply to the fractional integral operators and the generalized fractional integral operators.

This paper is organized as follows. Section 2 presents some notions and definitions for the subsequent sections. The main results on the boundedness of vector-valued operators on Morrey spaces and block spaces, Theorems 3.1 and 3.2, are established in Section 3. Section 3 also gives some applications of our main results on the Fefferman-Stein vector-valued inequalities and the mapping properties of the fractional integral operators and the generalized fractional integral operators. Finally, we introduce and study the Triebel-Lizorkin block spaces in Section 4.

\section{Definitions and preliminaries}

For any $x \in \mathbb{R}^{n}$ and $r>0$, let $B(x, r)=\left\{y \in \mathbb{R}^{n}:|x-y|<r\right\}$, and let $\mathbb{B}=$ $\left\{B\left(x_{0}, r\right): x_{0} \in \mathbb{R}^{n}, r>0\right\}$. Let $\mathcal{M}$ and $L_{\text {loc }}$ denote the space of Lebesgue measurable functions and the space of locally integrable functions on $\mathbb{R}^{n}$, respectively. Let $\mathrm{M}$ denote the Hardy-Littlewood maximal operator. Let $\mathcal{S}\left(\mathbb{R}^{n}\right)$ and $\mathcal{S}^{\prime}\left(\mathbb{R}^{n}\right)$ denote the class of Schwartz functions and tempered distributions, respectively. In addition,

$$
\mathcal{S}_{0}\left(\mathbb{R}^{n}\right)=\left\{f \in \mathcal{S}\left(\mathbb{R}^{n}\right): \int_{\mathbb{R}^{n}} x^{\gamma} f(x) d x=0, \forall \gamma \in \mathbb{N}^{n}\right\} .
$$

Let $\mathcal{P}$ denote the class of polynomials on $\mathbb{R}^{n}$.

We recall the definition of a Banach function space (BFS) (see [6, Chapter 1, Definitions 1.1 and 1.3]).

\section{DEFINITION 2.1}

A Banach space $X \subset \mathcal{M}$ is said to be a BFS if it satisfies

(a) $\|f\|_{X}=0 \Leftrightarrow f=0$ a.e.;

(b) $|g| \leq|f|$ a.e. $\Rightarrow\|g\|_{X} \leq\|f\|_{X}$;

(c) $0 \leq f_{n} \uparrow f$ a.e. $\Rightarrow\left\|f_{n}\right\|_{X} \uparrow\|f\|_{X}$;

(d) $\chi_{E} \in \mathcal{M}$ and $|E|<\infty \Rightarrow \chi_{E} \in X$;

(e) $\chi_{E} \in \mathcal{M}$ and $|E|<\infty \Rightarrow \int_{E}|f(x)| d x<C_{E}\|f\|_{X}, \forall f \in X$,

for some $C_{E}>0$. 
For any BFS $X$, let $X^{\prime}$ denote the associate space of $X$ (see [6, Chapter 1 , Definitions 2.1 and 2.3], [45, Volume II, p. 29]).

Let $\mathcal{Q}$ denote the family of cubes in $\mathbb{R}^{n}$ with sides parallel to the coordinate axes. For any $Q \in \mathcal{Q}$, let $l(Q)$ and $|Q|$ denote its side length and Lebesgue measure, respectively.

\section{DEFINITION 2.2}

For any $\rho:[0, \infty) \rightarrow[0, \infty)$, we write $\rho \in \mathbb{D}$ if $t^{-n} \rho(t)$ is decreasing and $\rho$ satisfies the doubling condition. That is, there exists a constant $C>0$ such that

$$
\frac{1}{C} \leq \frac{\rho(s)}{\rho(t)} \leq C, \quad \frac{1}{2} \leq \frac{s}{t} \leq 2
$$

For any $\rho \in \mathbb{D}$, the generalized fractional maximal operator is defined by

$$
\left(\mathrm{M}_{\rho} f\right)(x)=\sup _{B \ni x} \frac{\rho\left(|B|^{1 / n}\right)}{|B|} \int_{B}|f(y)| d y, \quad f \in L_{\mathrm{loc}},
$$

where the supremum is taken over all balls $B$ containing $x$.

For any $0 \leq \alpha<n$, when $\rho_{\alpha}(t)=t^{\alpha}$, the generalized fractional maximal operator $\mathrm{M}_{\rho_{\alpha}}$ becomes the fractional maximal operator

$$
\left(\mathrm{M}_{\alpha} f\right)(x)=\sup _{B \ni x} \frac{1}{|B|^{1-\alpha / n}} \int_{B}|f(y)| d y
$$

where the supremum is taken over all balls $B$ containing $x$. When $\alpha=0$, the fractional maximal operator reduces to the Hardy-Littlewood maximal operator M.

Furthermore, as $\rho \in \mathbb{D}$, the operator

$$
\left(\mathrm{M}_{\rho}^{\mathcal{Q}} f\right)(x)=\sup _{Q \ni x} \frac{\rho(l(Q))}{|Q|} \int_{Q}|f(y)| d y,
$$

where the supremum is taken over all cubes $Q$ containing $x$, is pointwise equivalent to $\mathrm{M}_{\rho}$. More precisely, we have a constant $C>0$ such that, for any $x \in \mathbb{R}^{n}$ and $f \in L_{\text {loc }}$, we have

$$
\frac{1}{C}\left(\mathrm{M}_{\rho} f\right)(x) \leq\left(\mathrm{M}_{\rho}^{\mathcal{Q}} f\right)(x) \leq C\left(\mathrm{M}_{\rho} f\right)(x) .
$$

We now introduce a new notion about the mapping property of $\mathrm{M}_{\rho}$ on BFS.

\section{DEFINITION 2.3}

Let $\rho \in \mathbb{D}$. Let $X$ and $X_{\rho}$ be BFSs. We call $\left(X, X_{\rho}\right)$ a $\rho$-Riesz pair if $\mathrm{M}_{\rho}: X \rightarrow X_{\rho}$ is bounded.

For simplicity, when $\rho_{\alpha}(t)=t^{\alpha}$ with $0 \leq \alpha<n$, we use the term $\alpha$-Riesz pair to describe a $\rho_{\alpha}$-Riesz pair. We now present a crucial result for $\rho$-Riesz pairs.

PROPOSITION 2.1

Let $\rho \in \mathbb{D}$, and let $X, X_{\rho}$ be BFS. If $\left(X, X_{\rho}\right)$ is a $\rho$-Riesz pair, then there exists 
a constant $C>0$ such that, for any $B \in \mathbb{B}$,

$$
\left\|\chi_{B}\right\|_{X^{\prime}}\left\|\chi_{B}\right\|_{X_{\rho}} \leq C \frac{|B|}{\rho\left(|B|^{1 / n}\right)} .
$$

Proof

We consider the operator $P_{B, \rho}(g), B=B\left(x_{0}, r\right), x_{0} \in \mathbb{R}^{n}$, and $r>0$, defined by

$$
\left(P_{B, \rho} g\right)(y)=\left(\frac{\rho\left(|B|^{1 / n}\right)}{|B|} \int_{B}|g(x)| d x\right) \chi_{B}(y) .
$$

The operator $P_{B, \rho}$ is uniformly dominated by the generalized fractional maximal operator $\mathrm{M}_{\rho}$. That is, there exists a constant $C>0$ such that, for any $B=$ $B\left(x_{0}, r\right), P_{B, \rho}(g) \leq \mathrm{M}_{\rho}(g)$. Hence, $\sup _{B}\left\|P_{B, \rho}\right\|_{X \rightarrow X_{\rho}}<C\left\|\mathrm{M}_{\rho}\right\|_{X \rightarrow X_{\rho}}$.

The uniform boundedness of $P_{B, \rho}$ and [6, Chapter 1, Theorem 2.9] show that

$$
\begin{aligned}
& \left\|\chi_{B}\right\|_{X^{\prime}}\left\|\chi_{B}\right\|_{X_{\rho}} \\
& =\sup \left\{\left|\int_{B} g d x\right|\left\|\chi_{B}\right\|_{X_{\rho}}: g \in X,\|g\|_{X} \leq 1\right\} \\
& \quad=\sup \left\{\frac{\rho\left(|B|^{1 / n}\right)}{|B|}\left|\int_{B} g d x\right|\left\|\chi_{B}\right\|_{X_{\rho}}: g \in X,\|g\|_{X} \leq 1\right\} \frac{|B|}{\rho\left(|B|^{1 / n}\right)} \\
& \quad \leq C \sup \left\{\left\|P_{B, \rho}(g)\right\|_{X_{\rho}}: g \in X,\|g\|_{X} \leq 1\right\} \frac{|B|}{\rho\left(|B|^{1 / n}\right)} \\
& \quad \leq C \frac{|B|}{\rho\left(|B|^{1 / n}\right)} .
\end{aligned}
$$

The main results of this paper, Theorems 3.1 and 3.2, rely on the above proposition.

\section{DEFINITION 2.4}

Let $X$ be a BFS. We write $X \in \mathbb{M}$ if the Hardy-Littlewood maximal operator $\mathrm{M}$ is bounded on $X$. We write $X \in \mathbb{M}^{\prime}$ if $X^{\prime} \in \mathbb{M}$.

We have a similar result when $X \in \mathbb{M} \cup \mathbb{M}^{\prime}$.

\section{LEMMA 2.2}

Let $X$ be a BFS. If $X \in \mathbb{M} \cup \mathbb{M}^{\prime}$, then there is a constant $C \geq 1$ such that

$$
|B| \leq\left\|\chi_{B}\right\|_{X}\left\|\chi_{B}\right\|_{X^{\prime}} \leq C|B|, \quad \forall B \in \mathbb{B} .
$$

The proof is similar to the proof of Proposition 2.1. For details, the reader is referred to [34, Lemma 3.2]. The above lemma also generalizes the corresponding result in [40].

The following definition was given in [35, Definition 2.2]. It is inspired by the family of sequence spaces $\left\{l_{\nu}^{q(x)}\right\}_{x \in \mathbb{R}^{n}}$ introduced in [17, p. 1737]. 


\section{DEFINITION 2.5}

A family of Banach lattices $\mathcal{B}=\{\mathcal{B}(x)\}_{x \in \mathbb{R}^{n}}$ is called a family of variable Banach sequence spaces (VBSs) if $\mathcal{B}(x) \subset\left\{\left\{a_{i}\right\}_{i \in \mathbb{Z}}: a_{i} \in \mathbb{C}\right\}$ and there exists a constant $C>0$ independent of $x \in \mathbb{R}^{n}$ such that, for any $k \in \mathbb{Z}$,

$$
\left|a_{k}\right| \leq C\left\|\left\{a_{i}\right\}_{i \in \mathbb{Z}}\right\|_{\mathcal{B}(x)}, \quad \forall x \in \mathbb{R}^{n} .
$$

For any VBS $\mathcal{B}=\{\mathcal{B}(x)\}_{x \in \mathbb{R}^{n}}$, denote the class of $\mathcal{B}$-valued Lebesgue measurable functions by $\mathcal{M}(\mathcal{B})$. More precisely,

$$
\mathcal{M}(\mathcal{B})=\left\{f=\left\{f_{i}\right\}_{i \in \mathbb{Z}}: f_{i}(x) \text { and }\|f(x)\|_{\mathcal{B}(x)} \in \mathcal{M}\right\} .
$$

Let $\mathcal{B}$ be a VBS. Whenever $X$ is a BFS, define

$$
X(\mathcal{B})=\left\{f \in \mathcal{M}(\mathcal{B}):\|\| f(x)\left\|_{\mathcal{B}(x)}\right\|_{X}<\infty\right\}
$$

and write

$$
\|f\|_{X(\mathcal{B})}=\|\| f(x)\left\|_{\mathcal{B}(x)}\right\|_{X} .
$$

We give the definition of the vector-valued Morrey spaces associated with BFS in the following.

\section{DEFINITION 2.6}

Let $X$ be a BFS, and let $\mathcal{B}$ be a VBS. Let $u(x, r): \mathbb{R}^{n} \times(0, \infty) \rightarrow(0, \infty)$ be a Lebesgue measurable function. The vector-valued Morrey space $\mathcal{M}_{u}^{X}(\mathcal{B})$ is the collection of all $f \in \mathcal{M}(\mathcal{B})$ satisfying

$$
\|f\|_{\mathcal{M}_{u}^{X}(\mathcal{B})}=\sup _{z \in \mathbb{R}^{n}, R>0} \frac{1}{u(z, R)}\left\|\chi_{B(z, R)} f\right\|_{X(\mathcal{B})}<\infty .
$$

The introduction of the function $u$ in the above definition is motivated by the examples presented in [21]. For the scalar-valued Morrey space, we write $\mathcal{M}_{u}^{X}(\mathbb{C})$ by $\mathcal{M}_{u}^{X}$.

We now introduce the vector-valued block spaces associated with BFSs. We have several equivalent definitions for the classical block spaces (see [2], [1], [27], [42], [77]). The following uses the notion of block to define our function space because it can be easily generalized to block spaces associated with BFSs.

\section{DEFINITION 2.7}

Let $X$ be a BFS, and let $\mathcal{B}$ be a VBS. Let $u(x, r): \mathbb{R}^{n} \times(0, \infty) \rightarrow(0, \infty)$ be a Lebesgue measurable function. A $b \in \mathcal{M}(\mathcal{B})$ is a $(u, X(\mathcal{B}))$-block if it is supported in a ball $B\left(x_{0}, r\right), x_{0} \in \mathbb{R}^{n}, r>0$, and

$$
\|b\|_{X(\mathcal{B})} \leq \frac{1}{u\left(x_{0}, r\right)} .
$$

Define the vector-valued block space $\mathfrak{B}_{u, X(\mathcal{B})}$ by

$$
\mathfrak{B}_{u, X(\mathcal{B})}=\left\{\sum_{k=1}^{\infty} \lambda_{k} b_{k}: \sum_{k=1}^{\infty}\left|\lambda_{k}\right|<\infty \text { and } b_{k} \text { is a }(u, X(\mathcal{B})) \text {-block }\right\} .
$$


The space $\mathfrak{B}_{u, X(\mathcal{B})}$ is endowed with the norm

$$
\|f\|_{\mathfrak{B}_{u, X(\mathcal{B})}}=\inf \left\{\sum_{k=1}^{\infty}\left|\lambda_{k}\right| \text { such that } f=\sum_{k=1}^{\infty} \lambda_{k} b_{k}\right\} .
$$

We write $\mathfrak{B}_{u, X}(\mathbb{C})$ by $\mathfrak{B}_{u, X}$. Next, we show that the block space $\mathfrak{B}_{u, X(\mathcal{B})}$ is a Banach lattice.

\section{PROPOSITION 2.3}

Let $X$ be a BFS, and let $\mathcal{B}$ be a VBS. Let $u(x, r): \mathbb{R}^{n} \times(0, \infty) \rightarrow(0, \infty)$ be a Lebesgue measurable function. Then $\mathfrak{B}_{u, X(\mathcal{B})}$ is a Banach lattice.

\section{Proof}

Obviously, $\|\cdot\|_{\mathfrak{B}_{u, X(\mathcal{B})}}$ satisfies the triangle inequality. Let $h_{j} \in \mathfrak{B}_{u, X(\mathcal{B})}, j \in \mathbb{N}$, satisfy

$$
\sum_{j=1}^{\infty}\left\|h_{j}\right\|_{\mathfrak{B}_{u, X(\mathcal{B})}}<\infty .
$$

According to the definition of $\mathfrak{B}_{u, X(\mathcal{B})}$, for any $\epsilon>0$, we have

$$
h_{j}=\sum_{k=1}^{\infty} \lambda_{k, j} b_{k, j}
$$

where $b_{k, j}, j, k \in \mathbb{N}$ are $(u, X(\mathcal{B}))$-blocks and

$$
\sum_{k=1}^{\infty}\left|\lambda_{k, j}\right| \leq(1+\epsilon)\left\|h_{j}\right\|_{\mathfrak{B}_{u, X(\mathcal{B})}} .
$$

Therefore,

$$
\sum_{j=1}^{\infty} h_{j}=\sum_{j=1}^{\infty} \sum_{k=1}^{\infty} \lambda_{k, j} b_{k, j}
$$

and $\lambda_{k, j}, j, k \in \mathbb{N}$, satisfy

$$
\sum_{j=1}^{\infty} \sum_{k=1}^{\infty}\left|\lambda_{k, j}\right| \leq(1+\epsilon) \sum_{j=1}^{\infty}\left\|h_{j}\right\|_{\mathfrak{B}_{u, X(\mathcal{B})}}<\infty .
$$

That is, $\sum_{j=1}^{\infty} h_{j}$ converges in $\mathfrak{B}_{u, X(\mathcal{B})}$. Moreover, as $\epsilon>0$ is arbitrary, we also have

$$
\left\|\sum_{j=1}^{\infty} h_{j}\right\|_{\mathfrak{B}_{u, X(\mathcal{B})}} \leq \sum_{j=1}^{\infty}\left\|h_{j}\right\|_{\mathfrak{B}_{u, X(\mathcal{B})}} .
$$

Hence, $\mathfrak{B}_{u, X(\mathcal{B})}$ is a Banach space.

Next, assume that $|g| \leq|f|$ where $f=\left\{f_{m}\right\}_{m \in \mathbb{Z}} \in \mathfrak{B}_{u, X(\mathcal{B})}$ and $g=\left\{g_{m}\right\}_{m \in \mathbb{Z}} \in$ $\mathcal{M}(\mathcal{B})$. Note that the ordering $|g| \leq|f|$ means that $\left|g_{m}\right| \leq\left|f_{m}\right|, \forall m \in \mathbb{Z}$. 
Since $f \in \mathfrak{B}_{u, X(\mathcal{B})}$, for any $\epsilon>0$, we have a family of $(u, X(\mathcal{B}))$-blocks $\left\{b_{i}\right\}_{i=1}^{\infty}$, $b_{i}=\left\{b_{i}^{m}\right\}_{m \in \mathbb{Z}}$, and a family of scalars $\left\{\lambda_{i}\right\}_{i=1}^{\infty}$ such that

$$
f=\sum_{i=1}^{\infty} \lambda_{i} b_{i}=\left\{f_{m}\right\}_{m \in \mathbb{Z}}=\left\{\sum_{i=1}^{\infty} \lambda_{i} b_{i}^{m}\right\}_{m \in \mathbb{Z}}
$$

and $\sum_{i=1}^{\infty}\left|\lambda_{i}\right| \leq(1+\epsilon)\|f\|_{\mathfrak{B}_{u, X(\mathcal{B})}}$. Therefore

$$
g=\sum_{i=1}^{\infty} \lambda_{i} c_{i}=\left\{g_{m}\right\}_{m \in \mathbb{Z}}=\left\{\sum_{i=1}^{\infty} \lambda_{i} c_{i}^{m}\right\}_{m \in \mathbb{Z}},
$$

where $c_{i}=\left\{c_{i}^{m}\right\}_{m \in \mathbb{Z}}$ and

$$
c_{i}^{m}(x)= \begin{cases}\frac{g_{m}(x)}{f_{m}(x)} b_{i}^{m}(x) & f_{m}(x) \neq 0, \\ 0 & f_{m}(x)=0 .\end{cases}
$$

It is easy to see that $\left\{c_{i}\right\}_{i=1}^{\infty}$ are $(u, X(\mathcal{B}))$-blocks because $\left|g_{m}\right| \leq\left|f_{m}\right|$, $\forall m \in \mathbb{Z}$. Thus, $g \in \mathfrak{B}_{u, X(\mathcal{B})}$. Moreover, as $\epsilon$ is arbitrary, we also have $\|g\|_{\mathfrak{B}_{u, X(\mathcal{B})}} \leq$ $\|f\|_{\mathfrak{B}_{u, X(\mathcal{B})}}$.

\section{Vector-valued operators with singular kernels}

The main results on vector-valued operators with singular kernel are presented in this section. Roughly speaking, the main results find that, whenever $\left(X, X_{\rho}\right)$ is a $\rho$-Riesz pair and an operator is bounded from $X$ to $X_{\rho}$, then this operator is also bounded from $\mathcal{M}_{u}^{X}$ to $\mathcal{M}_{u}^{X}$ and from $\mathfrak{B}_{u, X}$ to $\mathfrak{B}_{u, X_{\rho}}$.

We give the precise family of operators for which our main results apply in the following.

\section{DEFINITION 3.1}

Let $\rho \in \mathbb{D}$. Let $\mathcal{B}_{1}=\left\{\mathcal{B}_{1}(x)\right\}$ and $\mathcal{B}_{2}=\left\{\mathcal{B}_{2}(x)\right\}$ be VBSs. A sublinear operator $T: \mathcal{M}\left(\mathcal{B}_{1}\right) \rightarrow \mathcal{M}\left(\mathcal{B}_{2}\right)$ is called an operator with weakly singular kernel for $\left(\mathcal{B}_{1}, \mathcal{B}_{2}\right)$ if there exists a $C>0$ such that, for any $x \in \mathbb{R}^{n}$ and $f \in \mathcal{M}\left(\mathcal{B}_{1}\right)$ with $\operatorname{supp} f \subset$ $\mathbb{R}^{n} \backslash B(x, r)$, for some $r>0$,

$$
\|(T f)(x)\|_{\mathcal{B}_{2}(x)} \leq C \frac{\rho(r)}{r^{n}} \int\|f(y)\|_{\mathcal{B}_{1}(y)} d y .
$$

In addition, a linear operator $T: \mathcal{M}\left(\mathcal{B}_{1}\right) \rightarrow \mathcal{M}\left(\mathcal{B}_{2}\right)$ is said to be a linear operator with singular kernel for $\left(\mathcal{B}_{1}, \mathcal{B}_{2}\right)$ if there exists $K(x, y): \mathcal{B}_{1}(y) \rightarrow \mathcal{B}_{2}(x)$ such that

$$
T f(x)=\int K(x, y) f(y) d y, \quad \forall x \in \mathbb{R}^{n} \backslash \operatorname{supp} f,
$$

and

$$
\|K(x, y)\|_{\mathcal{B}_{1}(y) \rightarrow \mathcal{B}_{2}(x)} \leq C \frac{\rho(|x-y|)}{|x-y|^{n}}, \quad \forall(x, y) \in \mathbb{R}^{2 n} \backslash\left\{(z, z): z \in \mathbb{R}^{n}\right\},
$$

for some $C>0$. We call $K(x, y)$ the kernel of $T$.

Obviously, when $\rho \in \mathbb{D}$, a linear operator with singular kernel satisfies (3.1). In particular, when $\mathcal{B}_{1}(x)=\mathcal{B}_{2}(y)=\mathbb{R}, \forall x, y \in \mathbb{R}^{n}$, the above definition includes 
the singular operator with singular kernel studied in [72]. Moreover, it also covers the singular integral operator, the generalized fractional integral operators (see [63]), the fractional integral operators, and some important sublinear operators such as the Hardy-Littlewood maximal operator, the generalized fractional maximal operators (see [63]), and the fractional maximal operators.

We have another interesting example arising from the variable exponent analysis. Roughly speaking, when $p(x), q(x): \mathbb{R}^{n} \rightarrow(1, \infty)$ are locally log-Hölder continuous and globally log-Hölder continuous (see Definition 4.2), then the $\phi-\psi$ transforms defined in $L^{p(x)}\left(l^{q(x)}\right)$ are linear operators with singular kernel associated with $\mathcal{B}_{1}(x)=\mathcal{B}_{2}(x)=l^{q(x)}, x \in \mathbb{R}^{n}$. This result is presented and proved in Theorem 4.1. When $\rho_{\alpha}(t)=t^{\alpha}, 0 \leq \alpha<n$, an important example of operators with weakly singular kernel are the fractional integral operators

$$
\left(I_{\alpha} f\right)(x)=\int_{\mathbb{R}^{n}} \frac{f(y)}{|x-y|^{n-\alpha}} d y .
$$

The definitions of the above notions and details of these results are given in the following sections of this paper.

We now present and prove the main results of this paper. The first one is the boundedness of the operators with weakly singular kernel on block spaces.

\section{THEOREM 3.1}

Let $\rho \in \mathbb{D}$. Let $\left(X, X_{\rho}\right)$ be a $\rho$-Riesz pair, and let $u(x, r): \mathbb{R}^{n} \times(0, \infty) \rightarrow(0, \infty)$ be a Lebesgue measurable function. Let $\mathcal{B}_{1}=\left\{\mathcal{B}_{1}(x)\right\}$ and $\mathcal{B}_{2}=\left\{\mathcal{B}_{2}(x)\right\}$ be VBSs. Let $T$ be an operator with weakly singular kernel for $\left(\mathcal{B}_{1}, \mathcal{B}_{2}\right)$. If $T: X\left(\mathcal{B}_{1}\right) \rightarrow X_{\rho}\left(\mathcal{B}_{2}\right)$ is bounded and there exists a constant $C>0$ such that, for any $x \in \mathbb{R}^{n}$ and $r>0$, $u$ fulfills

$$
\begin{aligned}
u(x, 2 r) & \leq C u(x, r), \\
\sum_{j=0}^{\infty} \frac{\left\|\chi_{B(x, r)}\right\|_{X^{\prime}}}{\left\|\chi_{B\left(x, 2^{j+1} r\right)}\right\|_{X^{\prime}}} u\left(x, 2^{j+1} r\right) & <C u(x, r),
\end{aligned}
$$

then $T$ can be extended to be a bounded operator from $\mathfrak{B}_{u, X\left(\mathcal{B}_{1}\right)}$ to $\mathfrak{B}_{u, X_{\rho}\left(\mathcal{B}_{2}\right)}$.

Proof

Let $x_{0} \in \mathbb{R}^{n}, r>0$. Let $b$ be a $\left(u, X\left(\mathcal{B}_{1}\right)\right)$-block with support $B\left(x_{0}, r\right)$. Let $B_{0}=$ $B\left(x_{0}, 2 r\right)$ and $B_{k}=B\left(x_{0}, 2^{k} r\right), k \in \mathbb{N}$. Define $m_{k}=\chi_{B_{k+1} \backslash B_{k}} T(b)$, define $k \in$ $\mathbb{N} \backslash\{0\}$, and define $m_{0}=\chi_{B_{0}} T(b)$. Consequently, we have $\operatorname{supp} m_{k} \subseteq B_{k+1} \backslash B_{k}$ and $T(b)=\sum_{k=0}^{\infty} m_{k}$.

As $T$ is a bounded operator from $X\left(\mathcal{B}_{1}\right)$ to $X_{\rho}\left(\mathcal{B}_{2}\right)$, we have

$$
\begin{aligned}
\left\|m_{0}\right\|_{X_{\rho}\left(\mathcal{B}_{2}\right)} & \leq C\|T(b)\|_{X_{\rho}\left(\mathcal{B}_{2}\right)} \leq C\|b\|_{X\left(\mathcal{B}_{1}\right)} \\
& \leq \frac{C}{u\left(x_{0}, r\right)} \leq \frac{C}{u\left(x_{0}, 2 r\right)}
\end{aligned}
$$

for some constant $C>0$ independent of $x_{0}$ and $r$. We apply (3.2) for the last inequality in (3.4). Thus, $m_{0}$ is a constant multiple of a $\left(u, X_{\rho}\left(\mathcal{B}_{2}\right)\right)$-block. 
As $\operatorname{supp} b \subseteq B_{0}=B\left(x_{0}, r\right)$ and $\operatorname{supp} m_{k} \subseteq B_{k+1} \backslash B_{k}$, for any $x \in B_{k+1} \backslash B_{k}$, we find that $\operatorname{supp} b=B_{0} \subseteq \mathbb{R}^{n} \backslash B\left(x, 2^{k-1} r\right)$. Consequently, (3.1) shows that

$$
\begin{aligned}
\left\|m_{k}\right\|_{\mathcal{B}_{2}(x)} & =\chi_{B_{k+1} \backslash B_{k}}(x)\|T(b)(x)\|_{\mathcal{B}_{2}(x)} \\
& \leq C \chi_{B_{k+1} \backslash B_{k}}(x) \frac{\rho\left(2^{k} r\right)}{2^{k n} r^{n}} \int_{B\left(x_{0}, r\right)}\|b(y)\|_{\mathcal{B}_{1}(y)} d y,
\end{aligned}
$$

where we use (2.1) for the last inequality.

The Hölder inequality for $X$ (see [6, Chapter 1, Theorem 2.4]) ensures that

$$
\left\|m_{k}\right\|_{\mathcal{B}_{2}(x)} \leq C \chi_{B_{k+1} \backslash B_{k}}(x) \frac{\rho\left(2^{k} r\right)}{2^{k n} r^{n}}\|b\|_{X\left(\mathcal{B}_{1}\right)}\left\|\chi_{B\left(x_{0}, r\right)}\right\|_{X^{\prime}}, \quad \forall x \in \mathbb{R}^{n},
$$

for some $C>0$ independent of $k \in \mathbb{N}$ and $x \in \mathbb{R}^{n}$. Since $\left(X, X_{\rho}\right)$ is a $\rho$-Riesz pair, Proposition 2.1 with $B=B_{k+1}$ yields

$$
\left\|\chi_{B_{k+1}}\right\|_{X_{\rho}} \frac{\rho\left(2^{k+1} r\right)}{\left|B_{k+1}\right|} \leq C \frac{1}{\left\|\chi_{B_{k+1}}\right\|_{X^{\prime}}} .
$$

We apply the norm $\|\cdot\|_{X_{\rho}}$ on both sides of (3.5). Thus, (2.1) and the preceding inequality assert that

$$
\begin{aligned}
\left\|m_{k}\right\|_{X_{\rho}\left(\mathcal{B}_{2}\right)} & \leq C\left\|\chi_{B_{k+1} \backslash B_{k}}\right\|_{X_{\rho}} \frac{\rho\left(2^{k+1} r\right)}{\left|B_{k+1}\right|}\|b\|_{X\left(\mathcal{B}_{1}\right)}\left\|\chi_{B\left(x_{0}, r\right)}\right\|_{X^{\prime}} \\
& \leq C \frac{\left\|\chi_{B\left(x_{0}, r\right)}\right\|_{X^{\prime}}}{\left\|\chi_{B_{k+1}}\right\|_{X^{\prime}}} \frac{u\left(x_{0}, 2^{k+1} r\right)}{u\left(x_{0}, r\right)} \frac{1}{u\left(x_{0}, 2^{k+1} r\right)} .
\end{aligned}
$$

Write $m_{k}=\sigma_{k} b_{k}$, where

$$
\sigma_{k}=\frac{\left\|\chi_{B\left(x_{0}, r\right)}\right\|_{X^{\prime}}}{\left\|\chi_{B_{k+1}}\right\|_{X^{\prime}}} \frac{u\left(x_{0}, 2^{k+1} r\right)}{u\left(x_{0}, r\right)} .
$$

Hence, $b_{k}$ is a constant multiple of a $\left(u, X_{\rho}\left(\mathcal{B}_{2}\right)\right)$-block, and this constant does not depend on $k$. Inequality (3.3) guarantees that $\sum_{k=0}^{\infty} \sigma_{k}<C$ for some $C>0$. Therefore, $T(b) \in \mathfrak{B}_{u, X_{\rho}\left(\mathcal{B}_{2}\right)}$ and there exists a constant $C_{0}>0$ so that, for any $\left(u, X_{\rho}\left(\mathcal{B}_{2}\right)\right)$-block $b$,

$$
\|T(b)\|_{\mathfrak{B}_{u, X_{\rho}\left(\mathcal{B}_{2}\right)}}<C_{0} .
$$

Now, we consider $f \in \mathfrak{B}_{u, X\left(\mathcal{B}_{1}\right)}$. The definition of block space ensures that there exist a family of $\left(u, X\left(\mathcal{B}_{1}\right)\right)$-blocks $\left\{c_{k}\right\}_{k=1}^{\infty}$ and a sequence $\Lambda=\left\{\lambda_{k}\right\}_{k=1}^{\infty} \in l^{1}$ such that $f=\sum_{k=1}^{\infty} \lambda_{k} c_{k}$ with $\|\Lambda\|_{l^{1}} \leq 2\|f\|_{\mathfrak{B}_{u, X\left(\mathcal{B}_{1}\right)}}$. Finally, (3.6) yields

$$
\begin{aligned}
\|T(f)\|_{\mathfrak{B}_{u, X_{\rho}\left(\mathcal{B}_{2}\right)}} & \leq \sum_{k=1}^{\infty}\left|\lambda_{k}\right|\left\|T\left(c_{k}\right)\right\|_{\mathfrak{B}_{u, X_{\rho}\left(\mathcal{B}_{2}\right)}} \\
& \leq C_{0} \sum_{k=1}^{\infty}\left|\lambda_{k}\right| \leq 2 C_{0}\|f\|_{\mathfrak{B}_{u, X\left(\mathcal{B}_{1}\right)}} .
\end{aligned}
$$

Another main result of this paper is the boundedness of operators with weakly singular kernel on Morrey spaces. 


\section{THEOREM 3.2}

Let $\rho \in \mathbb{D}$. Suppose that $\left(X, X_{\rho}\right)$ is a $\rho$-Riesz pair, and suppose that $u(x, r)$ : $\mathbb{R}^{n} \times(0, \infty) \rightarrow(0, \infty)$ is a Lebesgue measurable function. Let $\mathcal{B}_{1}=\left\{\mathcal{B}_{1}(x)\right\}$ and $\mathcal{B}_{2}=\left\{\mathcal{B}_{2}(x)\right\}$ be VBSs. Let $T$ be an operator with weakly singular kernel for $\left(\mathcal{B}_{1}, \mathcal{B}_{2}\right)$.

If $T: X\left(\mathcal{B}_{1}\right) \rightarrow X_{\rho}\left(\mathcal{B}_{2}\right)$ is bounded and there exists a constant $C>0$ such that, for any $x \in \mathbb{R}^{n}$ and $r>0$, $u$ fulfills

$$
\begin{aligned}
u(x, 2 r) & \leq C u(x, r), \\
\sum_{j=0}^{\infty} \frac{\left\|\chi_{B(x, r)}\right\|_{X_{\rho}}}{\left\|\chi_{B\left(x, 2^{j+1} r\right)}\right\|_{X_{\rho}}} u\left(x, 2^{j+1} r\right) & <C u(x, r),
\end{aligned}
$$

then $T$ can be extended to be a bounded operator from $\mathcal{M}_{u}^{X}\left(\mathcal{B}_{1}\right)$ to $\mathcal{M}_{u}^{X_{\rho}}\left(\mathcal{B}_{2}\right)$.

Proof

Let $f \in \mathcal{M}_{u}^{X}\left(\mathcal{B}_{1}\right)$. For any $z \in \mathbb{R}^{n}$ and $r>0$, write $f(x)=f_{0}(x)+\sum_{j=1}^{\infty} f_{j}(x)$, where $f_{0}=\chi_{B(z, 2 r)} f$ and $f_{j}=\chi_{B\left(z, 2^{j+1} r\right) \backslash B\left(z, 2^{j} r\right)} f, j \in \mathbb{N}$. As $T: X\left(\mathcal{B}_{1}\right) \rightarrow X_{\rho}\left(\mathcal{B}_{2}\right)$ is bounded, we have $\left\|T\left(f_{0}\right)\right\|_{X_{\rho}\left(\mathcal{B}_{2}\right)} \leq C\left\|f_{0}\right\|_{X\left(\mathcal{B}_{1}\right)}$. Thus, we find that

$$
\begin{aligned}
\frac{1}{u(z, r)}\left\|\chi_{B(z, r)} T\left(f_{0}\right)\right\|_{X_{\rho}\left(\mathcal{B}_{2}\right)} & \leq C \frac{1}{u(z, 2 r)}\left\|\chi_{B(z, 2 r)} f\right\|_{X\left(\mathcal{B}_{1}\right)} \\
& \leq C \sup _{\substack{y \in \mathbb{R}^{n} \\
R>0}} \frac{1}{u(y, R)}\left\|\chi_{B(y, R)} f\right\|_{X\left(\mathcal{B}_{1}\right)}
\end{aligned}
$$

because (3.7) asserts that $u(z, 2 r)<C u(z, r)$ for some constant $C>0$ independent of $z \in \mathbb{R}^{n}$ and $r>0$.

According to the definition of operator with weakly singular kernel and (2.1), there is a constant $C>0$ such that, for any $j \geq 1$,

$$
\begin{aligned}
& \chi_{B(z, r)}(x)\left\|T\left(f_{j}\right)(x)\right\|_{\mathcal{B}_{2}(x)} \\
& \quad \leq C \frac{\rho\left(2^{j} r\right)}{2^{j n} r^{n}} \chi_{B(z, r)}(x) \int_{B\left(z, 2^{j+1} r\right)}\|f(y)\|_{\mathcal{B}_{1}(y)} d y .
\end{aligned}
$$

The Hölder inequality given in [6, Chapter 1, Theorem 2.4] ensures that

$$
\int_{B\left(z, 2^{j+1} r\right)}\|f(y)\|_{\mathcal{B}_{1}(y)} d y \leq\left\|\chi_{B\left(z, 2^{j+1} r\right)} f\right\|_{X\left(\mathcal{B}_{1}\right)}\left\|\chi_{B\left(z, 2^{j+1} r\right)}\right\|_{X^{\prime}}
$$

Subsequently, applying the norm $\|\cdot\|_{X_{\rho}}$ on both sides of (3.9), we have

$$
\begin{aligned}
\left\|\chi_{B(z, r)} T\left(f_{j}\right)\right\|_{X_{\rho}\left(\mathcal{B}_{2}\right)} \leq & C \frac{\rho\left(2^{j} r\right)}{2^{j n} r^{n}}\left\|\chi_{B(z, r)}\right\|_{X_{\rho}} \\
& \times\left\|\chi_{B\left(z, 2^{j+1} r\right)} f\right\|_{X\left(\mathcal{B}_{1}\right)}\left\|\chi_{B\left(z, 2^{j+1} r\right)}\right\|_{X^{\prime}}
\end{aligned}
$$

Proposition 2.1 guarantees that

$$
\left\|\chi_{B\left(x, 2^{j+1} r\right)}\right\|_{X^{\prime}} \frac{\rho\left(2^{j+1} r\right)}{\left|B_{j+1}\right|} \leq C \frac{1}{\left\|\chi_{B\left(x, 2^{j+1} r\right)}\right\|_{X_{\rho}}} .
$$


Therefore, (3.10) yields

$$
\left\|\chi_{B(z, r)} T\left(f_{j}\right)\right\|_{X_{\rho}\left(\mathcal{B}_{2}\right)} \leq C \frac{\left\|\chi_{B(z, r)}\right\|_{X_{\rho}}}{\left\|\chi_{B\left(z, 2^{j+1} r\right)}\right\| X_{X_{\rho}}}\left\|\chi_{B\left(z, 2^{j+1} r\right)} f\right\|_{X\left(\mathcal{B}_{1}\right)} .
$$

Thus,

$$
\begin{aligned}
& \left\|\chi_{B(z, r)} T\left(f_{j}\right)\right\|_{X_{\rho}\left(\mathcal{B}_{2}\right)} \\
& \quad \leq C \frac{\left\|\chi_{B(x, r)}\right\|_{X_{\rho}}}{\left\|\chi_{B\left(x, 2^{j+1} r\right)}\right\|_{X_{\rho}}} \frac{u\left(z, 2^{j+1} r\right)}{u\left(z, 2^{j+1} r\right)}\left\|\chi_{B\left(z, 2^{j+1} r\right)} f\right\|_{X\left(\mathcal{B}_{1}\right)} \\
& \quad \leq C \frac{\left\|\chi_{B(x, r)}\right\|_{X_{\rho}}}{\left\|\chi_{B\left(x, 2^{j+1} r\right)}\right\|_{X_{\rho}}} u\left(z, 2^{j+1} r\right) \sup _{\substack{y \in \mathbb{R}^{n} \\
R>0}} \frac{1}{u(y, R)}\left\|\chi_{B(y, R)} f\right\|_{X_{\rho}\left(\mathcal{B}_{2}\right)} .
\end{aligned}
$$

Furthermore, we obtain

$$
\begin{aligned}
\frac{1}{u(z, r)}\left\|\chi_{B(z, r)} T(f)\right\|_{X_{\rho}\left(\mathcal{B}_{2}\right)} & \leq \frac{1}{u(z, r)} \sum_{j=0}^{\infty}\left\|\chi_{B(z, r)} T\left(f_{j}\right)\right\|_{X_{\rho}\left(\mathcal{B}_{2}\right)} \\
& \leq C \sup _{\substack{y \in \mathbb{R}^{n} \\
R>0}} \frac{1}{u(y, R)}\left\|\chi_{B(y, R)} f\right\|_{X\left(\mathcal{B}_{1}\right)},
\end{aligned}
$$

where the constant $C>0$ is independent of $r$ and $z$. Finally, by taking the supremum over $z \in \mathbb{R}^{n}$ and $r>0$, we obtain the boundedness of $T: \mathcal{M}_{u}^{X}\left(\mathcal{B}_{1}\right) \rightarrow$ $\mathcal{M}_{u}^{X_{\rho}}\left(\mathcal{B}_{2}\right)$.

When the domain function space and the target function space of the operator $T$ are identical, the conditions given in Theorems 3.1 and 3.2 can be slightly relaxed.

\section{THEOREM 3.3}

Let $X \in \mathbb{M} \cup \mathbb{M}^{\prime}$, and let $u(x, r): \mathbb{R}^{n} \times(0, \infty) \rightarrow(0, \infty)$ be a Lebesgue measurable function. Suppose that $\mathcal{B}_{1}=\left\{\mathcal{B}_{1}(x)\right\}$ and $\mathcal{B}_{2}=\left\{\mathcal{B}_{2}(x)\right\}$ are VBSs. Let $T$ : $X\left(\mathcal{B}_{1}\right) \rightarrow X\left(\mathcal{B}_{2}\right)$ be a bounded operator with weakly singular kernel for $\left(\mathcal{B}_{1}, \mathcal{B}_{2}\right)$.

(a) If there exists a constant $C>0$ such that for any $x \in \mathbb{R}^{n}$ and $r>0$

$$
\sum_{j=0}^{\infty} \frac{\left\|\chi_{B(x, r)}\right\|_{X^{\prime}}}{\left\|\chi_{B\left(x, 2^{j+1} r\right)}\right\|_{X^{\prime}}} u\left(x, 2^{j+1} r\right)<C u(x, r),
$$

then $T$ can be extended to be a bounded operator on $\mathfrak{B}_{u, X(\mathcal{B})}$.

(b) If there exists a constant $C>0$ such that, for any $x \in \mathbb{R}^{n}$ and $r>0, u$ fulfills

$$
\sum_{j=0}^{\infty} \frac{\left\|\chi_{B(x, r)}\right\|_{X}}{\left\|\chi_{B\left(x, 2^{j+1} r\right)}\right\|_{X}} u\left(x, 2^{j+1} r\right)<C u(x, r),
$$

then $T$ can be extended to be a bounded operator on $\mathcal{M}_{u}^{X}(\mathcal{B})$. 
Proof

The proof of the above result is similar to the proofs of Theorems 3.1 and 3.2. For simplicity, we just outline the proof.

As $X \in \mathbb{M} \cup \mathbb{M}^{\prime}$, Lemma 2.2 yields

$$
\left\|\chi_{B(x, 2 r)}\right\|_{X} \leq \frac{|B(x, 2 r)|}{\left\|\chi_{B(x, 2 r)}\right\|_{X^{\prime}}} \leq C \frac{|B(x, r)|}{\left\|\chi_{B(x, r)}\right\|_{X^{\prime}}} \leq C\left\|\chi_{B(x, r)}\right\|_{X}
$$

for some $C>0$. Similarly, we also have $\left\|\chi_{B(x, 2 r)}\right\|_{X^{\prime}} \leq\left\|\chi_{B(x, r)}\right\|_{X^{\prime}}$. Therefore, (3.2) and (3.7) follow from (3.11) and (3.12), respectively.

The rest of the proof is the same as the proofs of Theorems 3.1 and 3.2; the only modification is replacing Proposition 2.1 by Lemma 2.2. For simplicity, we skip the details and leave it to the reader.

In fact, the boundedness results for some operators on the classical Morrey spaces, such as the singular integral operators, can be obtained from the boundedness of these operators on the classical block spaces since the dual spaces of the classical block spaces are the classical Morrey spaces.

On the other hand, this argument may not be valid for vector-valued function spaces. For instance, the dual space of the vector-valued Lebesgue space $L^{p}(\mathcal{B})=$ $\mathfrak{B}_{L^{p}(\mathcal{B}), u}, 1<p<\infty$, where $\mathcal{B}$ is a Banach lattice and $u \equiv 1$, is equal to $L^{p^{\prime}}\left(\mathcal{B}^{*}\right)=$ $\mathcal{M}_{u}^{L^{p^{\prime}}\left(\mathcal{B}^{*}\right)}$ when $p^{\prime}$ is the conjugate of $p$ and $\mathcal{B}^{*}$ has the Radon-Nikodym property [19]. Our main results do not need the Radon-Nikodym property for $\mathcal{B}_{1}^{*}$ and $\mathcal{B}_{2}^{*}$.

In the following, we present some applications of Theorems 3.1 and 3.2. We generalize the Fefferman-Stein vector-valued maximal inequalities. We also extend the mapping properties of the fractional integral operators and the generalized fractional integral operator on Morrey-type spaces and block-type spaces.

We study the boundedness of the vector-valued Hardy-Littlewood maximal operator, which is defined by

$$
\mathfrak{M}(f)=\left\{\mathrm{M}\left(f_{i}\right)\right\}_{i \in \mathbb{N}}, \quad f=\left\{f_{i}\right\}_{i \in \mathbb{N}} \subset L_{\mathrm{loc}} .
$$

We recall the notion of $p$-convexification for BFSs before the statement and proof of the boundedness of the vector-valued Hardy-Littlewood maximal operator.

Let $X$ be a Banach lattice. For any $1 \leq p<\infty$, the $p$-convexification of $X$, $X^{p}$, is defined by

$$
X^{p}=\left\{f:|f|^{p} \in X\right\} .
$$

We equip $X^{p}$ with the norm $\|f\|_{X^{p}}=\left\||f|^{p}\right\|_{X}^{1 / p}$. For a more complete account of the $p$-convexification, the reader may consult [45, Volume II, p. 53].

In the proof of the Fefferman-Stein vector-valued maximal inequalities on block spaces and Morrey spaces, we also use the notion of the Muckenhoupt $A_{p^{-}}$ class, $1 \leq p \leq \infty$. For the details of the $A_{p}$-class, the reader is referred to $[69$, Section V].

The following theorem establishes the Fefferman-Stein vector-valued maximal inequalities on block spaces and Morrey spaces. 


\section{THEOREM 3.4}

Let $1<p, q<\infty$. Let $u$ satisfy (3.2), and let $X \in \mathbb{M}^{\prime}$.

(a) If $u$ satisfies

$$
\sum_{j=0}^{\infty} \frac{\left\|\chi_{B(x, r)}\right\|_{\left(X^{p}\right)^{\prime}}}{\left\|\chi_{B\left(x, 2^{j+1} r\right)}\right\|_{\left(X^{p}\right)^{\prime}}} u\left(x, 2^{j+1} r\right)<C_{0} u(x, r)
$$

for some constant $C_{0}>0$, then we have

$$
\|\| \mathfrak{M}(f)\left\|_{l^{q}}\right\|_{\mathfrak{B}_{u, X} p} \leq C\|\| f\left\|_{l^{q}}\right\|_{\mathfrak{B}_{u, X} p}
$$

for some $C>0$.

(b) If $u$ satisfies

$$
\sum_{j=0}^{\infty} \frac{\left\|\chi_{B(x, r)}\right\|_{X^{p}}}{\left\|\chi_{B\left(x, 2^{j+1} r\right)}\right\|_{X^{p}}} u\left(x, 2^{j+1} r\right)<C u(x, r)
$$

for some constant $C_{1}>0$, then we have

$$
\|\| \mathfrak{M}(f)\left\|_{l^{q}}\right\|_{\mathcal{M}_{u}^{X p}} \leq C\|\| f\left\|_{l^{q}}\right\|_{\mathcal{M}_{u}^{X p}}
$$

for some $C>0$.

\section{Proof}

We apply Theorem 3.3 to obtain (3.13) and (3.14). Therefore, we first need to obtain the Fefferman-Stein vector-valued maximal inequalities on $X^{p}$,

$$
\|\| \mathfrak{M}(f)\left\|_{l^{q}}\right\|_{X^{p}} \leq C\|\| f\left\|_{l^{q}}\right\|_{X^{p}} .
$$

Note that, according to [5, Theorem 3.1], we have the $A_{p}$-weighted FeffermanStein vector-valued maximal inequalities.

In view of $X \in \mathbb{M}^{\prime}$, by using the Rubio de Francia method of extrapolation (see [15], [26], [58]-[60], in particular, [14, Theorem 4.6]), we have

$$
\|\| \mathfrak{M}(f)\left\|_{l^{q}}\right\|_{X^{p}} \leq C\|\| f\left\|_{l^{q}}\right\|_{X^{p}}
$$

where $\left\{f_{j}\right\} \subset L_{0}^{\infty}$ and $L_{0}^{\infty}$ denote the class of bounded functions with compacted supported. Inequality (3.15) is valid because $X^{p}$ satisfies Definition 2.1(c) if and only if $X$ does and $f \chi_{\{x \in B(0, n):|f(x)| \leq n\}} \uparrow f, n \in \mathbb{N}$.

Then, we show that the vector-valued maximal operator $\mathfrak{M}(f)=\left\{\mathrm{M} f_{j}\right\}_{j=1}^{\infty}$, $f=\left\{f_{j}\right\}_{j=1}^{\infty}$, is an operator with weakly singular kernel associated with $\mathcal{B}=l^{q}$ and $\rho \equiv 1$.

Let $x \in \mathbb{R}^{n}$, let $r>0$, and let $\operatorname{supp} f_{j}=\mathbb{R}^{n} \backslash B(x, r), j \in \mathbb{N}$. Since

$$
\operatorname{dist}\left(x, \mathbb{R}^{n} \backslash B(x, r)\right)=r,
$$

there is a constant $C>0$ such that, for any $j \in \mathbb{N}$,

$$
\left(\mathrm{M} f_{j}\right)(x) \leq C \frac{1}{r^{n}} \int_{\mathbb{R}^{n}}\left|f_{j}(y)\right| d y .
$$


As $l^{q}$ is a Banach lattice, we find that

$$
\|\mathfrak{M} f(x)\|_{l^{q}} \leq C \frac{1}{r^{n}} \int_{\mathbb{R}^{n}}\|f(y)\|_{l^{q}} d y .
$$

The above inequality guarantees that $\mathfrak{M}$ is an operator with weakly singular kernel. Consequently, (3.13) and (3.14) follow from Theorem 3.3.

With respect to the notions and terminologies introduced in [32] and [33], we find that $\left(l^{q}, \mathfrak{B}_{u, \mathbb{X}^{p}}\right)$ and $\left(l^{q}, \mathcal{M}_{u}^{\mathbb{X}^{p}}\right)$ are admissible pairs and the corresponding Triebel-Lizorkin-type spaces $\dot{F}_{\mathfrak{B}_{u, \mathbb{X}^{p}}^{q}}^{q}$ and $\dot{F}_{\mathcal{M}_{u}^{q p}}^{q}$ are well defined. In addition, the $\phi-\psi$ transforms are bounded on the corresponding sequence spaces and function spaces. The atomic and molecular decompositions are valid. For brevity, we leave the details to the reader.

The approach given in [33] and [38] relies on the validity of the FeffermanStein vector-valued maximal inequalities. Hedberg and Netrusov [31] also used the Fefferman-Stein vector-valued maximal inequalities to study function spaces. By applying the results from [31], we have the spectral synthesis and the Luzin approximation of the Triebel-Lizorkin spaces associated with $\left(l^{q}, \mathfrak{B}_{u, X^{p}}\right)$ and $\left(l^{q}, \mathcal{M}_{u}^{X^{p}}\right)$. The reader may consult [31] for details.

When $X=L^{r}, 1<r<\infty$, the $\dot{F}_{\mathcal{M}_{u}^{X}}^{q}$ 's become the Triebel-Lizorkin-Morrey spaces studied in [65] and [70]. Notice that there is another approach for studying the Triebel-Lizorkin spaces associated with $\left(l^{q}, \mathfrak{B}_{u, X^{p}}\right)$ and $\left(l^{q}, \mathcal{M}_{u}^{X^{p}}\right)$ that is independent of the Fefferman-Stein vector-valued maximal inequalities (see [44]). Particularly, when $X$ is the Lebesgue space with variable exponents $L^{p(\cdot)},(3.13)$ generalizes the boundedness result of the Hardy-Littlewood maximal operator on block spaces with variable exponents obtained in [9] to vector-valued inequalities.

The Fefferman-Stein vector-valued maximal inequalities on Morrey spaces with variable exponents were obtained in [39]. These inequalities were also used in [39] to establish the atomic decompositions of Hardy-Morrey spaces with variable exponents. The Hardy-Morrey spaces with variable exponents are generalizations of Hardy-Morrey spaces (see [36], [41], [61]) and Hardy spaces with variable exponents (see [52]).

Our main result also applies to fractional integral operators and generalized fractional integral operators. For instance, some mapping properties of Orlicz spaces are established in [56, Theorem 4.7] and [71, Theorem 2.8]; Theorems 3.1 and 3.2 give us the boundedness of the fractional integral operator on the Orlicz block spaces and the Orlicz-Morrey spaces, respectively.

Similarly, in view of [20, Theorem 3.6.10], Theorems 3.1 and 3.2 also offer the boundedness of the fractional integral operators on the Lorentz-Karamata block spaces and the Lorentz-Karamata-Morrey spaces, respectively. The reader may consult [20, Chapter 3] for the definition and properties of Lorentz-Karamata spaces. The preceding results extend the mapping properties for the fractional integral operators on the classical Morrey spaces (see [57]).

We also have the mapping properties for the fractional integral operators on Morrey spaces with variable exponents and block spaces with variable exponents. 
Since the results for the Morrey spaces with variable exponents were already established in [28], [29], [37], and [51], we only present the result for the block spaces with variable exponents in the following. Since we present an extension of the study of Lebesgue spaces with variable exponents in the next section, for simplicity, we refer the reader to Definition 4.1 for the definition of Lebesgue spaces with variable exponents.

\section{THEOREM 3.5}

Let $0 \leq \alpha<n$, and let $p(x): \mathbb{R}^{n} \rightarrow(1, \infty)$ be a Lebesgue measurable function such that $L^{p(\cdot)} \in \mathbb{M}$. If u satisfies (3.2) and (3.3) with $X=L^{p(\cdot)}$, then

$$
\left\|I_{\alpha}(f)\right\|_{\mathfrak{B}_{u, L} q(\cdot)} \leq C\|f\|_{\mathfrak{B}_{u, L^{p(\cdot)}}}
$$

for some $C>0$ where

$$
\frac{1}{p(x)}-\frac{1}{q(x)}=\frac{\alpha}{n}, \quad \text { a.e. on } \mathbb{R}^{n} .
$$

According to [8] and [12], the fractional integral operator $I_{\alpha}$ is bounded from $L^{p(\cdot)}$ to $L^{q(\cdot)}$. Thus, the above result is ensured by Theorem 3.1. Note that, in the above theorem, the exponent function $p$ is not required to be locally logHölder continuous.

Finally, Theorem 3.1 and 3.2 are also applied to the generalized fractional integral operators. For a detailed study of the generalized fractional integral operators, the reader is referred to [49], [50], [62], [63], and [64]. Particularly, the studies in [50], [62], [63], and [64] are on the mapping properties of the generalized fractional integral operators on Morrey-type spaces. Our main results, specifically Theorem 3.1, extend the mapping properties of the generalized fractional integral operators to block-type spaces.

\section{DEFINITION 3.2}

Let $\rho \in \mathbb{D}$. The generalized fractional integral operator associated with $\rho$ is defined by

$$
I_{\rho}(f)(x)=\int_{\mathbb{R}^{n}} f(y) \frac{\rho(|x-y|)}{|x-y|^{n}} d y .
$$

THEOREM 3.6

Let $\rho \in \mathbb{D}$. Let $X$ and $X_{\rho}$ be BFSs. Suppose that there exists a constant $C>0$ such that

$$
\left\|I_{\rho}(f)\right\|_{X_{\rho}} \leq C\|f\|_{X}, \quad \forall f \in X
$$

(a) If $u$ satisfies (3.2) and (3.3), then

$$
\left\|I_{\rho}(f)\right\|_{\mathfrak{B}_{u, X}} \leq C\|f\|_{\mathfrak{B}_{u, X}}, \quad \forall f \in \mathfrak{B}_{u, X},
$$

for some $C>0$. 
(b) If $u$ satisfies (3.7) and (3.8), then

$$
\left\|I_{\rho}(f)\right\|_{\mathcal{M}_{u}^{X}} \leq C\|f\|_{\mathcal{M}_{u}^{X}}, \quad \forall f \in \mathcal{M}_{u}^{X},
$$

for some $C>0$.

Since $\mathrm{M}_{\rho}(f) \leq I_{\rho}(f)$ for any nonnegative locally integrable function $f$, the above results follow from Theorems 3.1 and 3.2. For some examples of function spaces $X, X_{\rho}$ for which (3.16) is fulfilled, the reader may consult [50, Examples 7.2 and $7.3]$.

Finally, Theorems 3.1 and 3.2 also apply to the generalized fractional maximal operators $\mathrm{M}_{\rho}$. For simplicity, we leave the details to the reader.

\section{Triebel-Lizorkin block spaces with variable exponents}

In this section, we define and study the Triebel-Lizorkin block spaces with variable exponents. Families of Besov spaces and Triebel-Lizorkin spaces associated with variable exponents were introduced in [4] and [17], respectively. Diening, Hästö, and Roudenko [17] even replaced the sequence space $l^{q}$ by $l_{\nu}^{q(x)}$ (see [17, p. 1737]). They found that the Fefferman-Stein vector-valued maximal inequalities are invalid in $L^{p(x)}\left(l^{q(x)}\right)$ (see [17, p. 1746]), and they established their results by considering some vector-valued singular integral operators on Lebesgue spaces with variable exponents.

Roughly speaking, the approach given in [17] relies on the boundedness of the vector-valued linear operator

$$
\mathcal{T}\left(\left\{f_{j}\right\}_{j \in \mathbb{N}}\right)=\left\{\varphi_{j} * f_{j}\right\}
$$

on $L^{p(x)}\left(l^{q(x)}\right)$, where $\varphi_{j}(x)=2^{n j}\left(1+2^{j}|x|\right)^{-m}$ for sufficiently large $m$. Thus, whenever we show that $\mathcal{T}$ is an operator with singular kernel, we can apply Theorem 3.3 to generalize the results in [17] to Morrey spaces with variable exponents and block spaces with variable exponents. In fact, this method had already been used in [35] for the study of Triebel-Lizorkin-Morrey spaces with variable exponents.

In this section, we use the boundedness of some vector-valued singular integral operators on block spaces with variable exponents to define and study the Triebel-Lizorkin block spaces with variable exponents. We begin with some notions and notations used in variable exponent analysis. For more complete and detailed results for Lebesgue spaces with variable exponents, the reader is referred to [11] and [16].

\section{DEFINITION 4.1}

Let $p(x): \mathbb{R}^{n} \rightarrow(0, \infty)$ be a Lebesgue measurable function. The Lebesgue space with variable exponent $L^{p(\cdot)}$ consists of all Lebesgue measurable functions $f$ : $\mathbb{R}^{n} \rightarrow \mathbb{C}$ so that 


$$
\|f\|_{L^{p(\cdot)}}=\inf \left\{\lambda>0: \int_{\mathbb{R}^{n}}\left|\frac{f(x)}{\lambda}\right|^{p(x)} d x \leq 1\right\}<\infty .
$$

We call $p(x)$ the exponent function of $L^{p(\cdot)}$.

The Lebesgue spaces with variable exponents were defined by Nakano [53], [54].

For any Lebesgue measurable function $p(x): \mathbb{R}^{n} \rightarrow(0, \infty)$, define $p_{-}=$ $\operatorname{essinf}_{x \in \mathbb{R}^{n}} p(x)$, and define $p_{+}=\operatorname{ess}_{\sup _{x \in \mathbb{R}^{n}}} p(x)$. Let $\mathbb{P}$ denote the class of exponent functions in which the Hardy-Littlewood maximal operator $M$ is bounded on $L^{p(\cdot)}$. The set $\mathbb{P}$ contains an important class of continuous functions.

\section{DEFINITION 4.2}

A continuous function $g: \mathbb{R}^{n} \rightarrow(0, \infty)$ is locally log-Hölder continuous if there exists $c_{\log }>0$ such that

$$
|g(x)-g(y)| \leq \frac{c_{\log }}{\log (e+1 /|x-y|)}, \quad \forall x, y \in \mathbb{R}^{n} .
$$

We denote the class of locally log-Hölder continuous functions by $C_{\operatorname{loc}}^{\log }\left(\mathbb{R}^{n}\right)$. Furthermore, a continuous function is globally log-Hölder continuous if $g \in C_{\operatorname{loc}}^{\log }\left(\mathbb{R}^{n}\right)$ and there exists $g_{\infty} \in \mathbb{R}$ so that

$$
\left|g(x)-g_{\infty}\right| \leq \frac{c_{\log }}{\log (e+1 /|x|)}, \quad \forall x \in \mathbb{R}^{n} .
$$

The class of globally log-Hölder continuous functions is denoted by $C^{\log }\left(\mathbb{R}^{n}\right)$.

For any $p \in C^{\log }\left(\mathbb{R}^{n}\right)$ with $1<p_{-} \leq p_{+}<\infty, p \in \mathbb{P}$ (see [13]). Note that $\mathbb{P} \backslash C^{\log }\left(\mathbb{R}^{n}\right)$ is nonempty; the reader may consult [55] for details.

We adopt the standing assumption introduced in [17] for the study of block spaces with variable exponents.

\section{DEFINITION 4.3}

The Lebesgue measurable functions $p(x), q(x): \mathbb{R}^{n} \rightarrow(0, \infty)$ and $\alpha(x): \mathbb{R}^{n} \rightarrow$ $[0, \infty)$ satisfy the standing assumptions if $p, q \in C^{\log }\left(\mathbb{R}^{n}\right)$ with $1<p_{-} \leq p_{+}<\infty$ and $1<q_{-} \leq q_{+}<\infty, \alpha \in C_{\operatorname{loc}}^{\log }\left(\mathbb{R}^{n}\right) \cap L^{\infty}$, and $\lim _{x \rightarrow \infty} \alpha(x)$ exists. We write $(p, q, \alpha) \in \mathbb{S}$ if they satisfy the standing assumptions.

Let $q(x) \in C^{\log }\left(\mathbb{R}^{n}\right)$ with $1<q_{-} \leq q_{+}<\infty$. For any family of Lebesgue measurable functions $\left\{f_{\nu}\right\}_{\nu \in \mathbb{N}}$, define

$$
\left\|f_{\nu}(x)\right\|_{l_{\nu}^{q(x)}}=\left(\sum_{\nu=0}^{\infty}\left|f_{\nu}(x)\right|^{q(x)}\right)^{1 / q(x)},
$$

and define $l_{\nu}^{q(\cdot)}=\left\{l_{\nu}^{q(x)}\right\}_{x \in \mathbb{R}^{n}}$. Moreover, let $\alpha$ be as in the standing assumptions. We define

$$
\left\|f_{\nu}(x)\right\|_{l_{\nu}^{\alpha(x), q(x)}}=\left(\sum_{\nu=0}^{\infty}\left(2^{\nu \alpha(x)}\left|f_{\nu}(x)\right|\right)^{q(x)}\right)^{1 / q(x)}
$$


and $l_{\nu}^{\alpha(\cdot), q(\cdot)}=\left\{l_{\nu}^{\alpha(x), q(x)}\right\}_{x \in \mathbb{R}^{n}}$. Whenever $1<q_{-} \leq q_{+}<\infty$ and $\alpha$ satisfies the standing assumptions, $l_{\nu}^{\alpha(\cdot), q(\cdot)}$ is a family of VBSs.

We are now ready to show that $\mathcal{T}$ is a linear operator with singular kernel for $\left(l_{\nu}^{\alpha(\cdot), q(\cdot)}, l_{\nu}^{\alpha(\cdot), q(\cdot)}\right)$. In fact, it follows from [35, Definition 5.3, Lemmas 5.1 and $6.8]$. For completeness, we present the proof in the following.

\section{THEOREM 4.1}

Let $q(\cdot) \in C^{\log }\left(\mathbb{R}^{n}\right)$ with $1<q_{-} \leq q_{+}<\infty$, let $\alpha \in C_{\operatorname{loc}}^{\log }\left(\mathbb{R}^{n}\right) \cap L^{\infty}$, and let $\lim _{x \rightarrow \infty} \alpha(x)$ exist. There exists a $\Gamma>0$ such that if

$$
\left|\phi_{\nu}(x)\right| \leq C 2^{n \nu}\left(1+2^{\nu}|x|\right)^{-n-\Gamma}, \quad \forall \nu \in \mathbb{N} \cup\{0\},
$$

then the vector-valued operator

$$
\mathcal{T}\left(\left\{f_{\nu}\right\}_{\nu \in \mathbb{N}}\right)=\left\{\phi_{\nu} * f_{\nu}\right\}
$$

is a linear operator with singular kernel for $\left(l_{\nu}^{\alpha(\cdot), q(\cdot)}, l_{\nu}^{\alpha(\cdot), q(\cdot)}\right)$.

Proof

We first show that there exist a constant $C$ independent of $x, y \in \mathbb{R}^{n}$ and a bounded nonnegative function $\gamma(x, y)$ so that, for any $x, y \in \mathbb{R}^{n}$ with $x \neq y$,

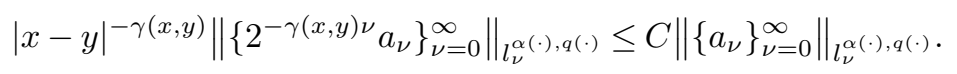

When $q(y) \leq q(x)$ and $\alpha(x) \leq \alpha(y)$, we have the embedding $l_{\nu}^{\alpha(y), q(y)} \hookrightarrow l_{\nu}^{\alpha(x), q(x)}$. Therefore, (4.2) is fulfilled with $\gamma(x, y)=0$.

If $q(x)<q(y)$, then Hölder's inequality guarantees that

$$
\left\|\left\{2^{-\nu / r(x, y)} a_{\nu}\right\}_{\nu=0}^{\infty}\right\|_{l_{\nu}^{q(x)}} \leq\left\|\left\{2^{-\nu /(r(x, y))}\right\}_{\nu=0}^{\infty}\right\|_{l_{\nu}^{r(x, y)}}\left\|\left\{a_{\nu}\right\}_{\nu=0}^{\infty}\right\|_{l_{\nu}^{q(y)}},
$$

where $1 / q(x)=1 /(r(x, y))+1 / q(y)$. Moreover, since $q \in C^{\log }\left(\mathbb{R}^{n}\right)$ with $1<q_{-} \leq$ $q_{+}<\infty$ implies $1 / q \in C^{\log }\left(\mathbb{R}^{n}\right)$, Definition 4.2 ensures that $|x-y|^{1 / q(y)-1 / q(x)}$ is bounded above. As $0<1 /(r(x, y)) \leq 1 / q_{-}-1 / q_{+}$, we have (4.2) for $l_{\nu}^{q(x)}$ with $\gamma(x, y)=1 /(r(x, y))=1 /(q(x))-1 /(q(y))$. Similarly, when $\alpha(y)<\alpha(x)$, we find that $|x-y|^{\alpha(y)-\alpha(x)}$ is bounded above in view of $\alpha \in C_{\operatorname{loc}}^{\log }\left(\mathbb{R}^{n}\right) \cap L^{\infty}\left(\mathbb{R}^{n}\right)$. Consequently, (4.2) is valid for $l_{\nu}^{\alpha(\cdot), q(\cdot)}$ with

$$
\begin{aligned}
\gamma(x, y)= & (\alpha(x)-\alpha(y)) \chi_{\left\{(x, y) \in \mathbb{R}^{2 n}: \alpha(y)<\alpha(x)\right\}} \\
& +\left(\frac{1}{q(x)}-\frac{1}{q(y)}\right) \chi_{\left\{(x, y) \in \mathbb{R}^{2 n}: q(x)<q(y)\right\}} .
\end{aligned}
$$

Write $\Gamma=\left[\left[\sup _{x, y \in \mathbb{R}^{n}} \gamma(x, y)\right]\right]+1$ where $[[\beta]]$ denotes the largest integer less than or equal to $\beta$. Fix $x, y \in \mathbb{R}^{n}$ with $x \neq y$. Let $U_{1}(y)=\left\{a \in l_{\nu}^{\alpha(y), q(y)}\right.$ : $\left.\|a\|_{l_{\nu}^{\alpha(y), q(y)}} \leq 1\right\}$. According to (4.1), the kernel of the operator $\mathcal{T}, K(x, y)$, satisfies

$$
\begin{aligned}
& \|K(x, y)\|_{l_{\nu}^{\alpha(y), q(y)} \rightarrow l_{\nu}^{\alpha(x), q(x)}} \\
& \quad \leq \sup _{\left\{a_{\nu}\right\}_{\nu=0}^{\infty} \in U_{1}(y)}\left\|\left\{\varphi_{j}(x-y) a_{\nu}\right\}_{\nu=0}^{\infty}\right\|_{l_{\nu}^{\alpha(x), q(x)}}
\end{aligned}
$$




$$
\begin{aligned}
& \leq C_{n+\Gamma} \sup _{\left\{a_{\nu}\right\}_{\nu=0}^{\infty} \in U_{1}(y)}\left\|\left\{\frac{2^{n \nu} a_{\nu}}{\left(1+2^{\nu}|x-y|\right)^{n+\gamma(x, y)}}\right\}_{\nu=0}^{\infty}\right\| \|_{l_{\nu}^{\alpha(x), q(x)}} \\
& \leq C \sup _{\left\{a_{\nu}\right\}_{\nu=0}^{\infty} \in U_{1}(y)}|x-y|^{-\gamma(x, y)-n}\left\|\left\{2^{-\gamma(x, y) \nu} a_{\nu}\right\}_{\nu=0}^{\infty}\right\|_{l_{\nu}^{\alpha(x), q(x)}} \\
& \leq C \frac{1}{|x-y|^{n}}
\end{aligned}
$$

for some $C>0$ independent of $x, y \in \mathbb{R}^{n}$ where we use (4.2) for the last inequality. Thus, $\mathcal{T}$ is a linear operator with singular kernel for $\left(l_{\nu}^{\alpha(\cdot), q(\cdot)}, l_{\nu}^{\alpha(\cdot), q(\cdot)}\right)$.

The reader may consult [35] for an application of the above result on the TriebelLizorkin-Morrey spaces with variable exponents.

We now use Theorem 4.1 to introduce and study some new function spaces. We are particularly interested in the Triebel-Lizorkin block spaces with variable exponents.

For any $f \in \mathcal{S}^{\prime}\left(\mathbb{R}^{n}\right)$, denote the Fourier transform of $f$ by $\hat{f}$.

\section{DEFINITION 4.4}

Let $(p, q, \alpha) \in \mathbb{S}$, and let $u$ be a Lebesgue measurable function satisfying (3.2) and (3.3). The Triebel-Lizorkin block space with variable exponent $F_{q(\cdot), \mathfrak{B}}^{\alpha(\cdot)}$ consists of those $f \in \mathcal{S}^{\prime}\left(\mathbb{R}^{n}\right)$ satisfying

$$
\|f\|_{F_{q(\cdot), \mathfrak{B}}^{\alpha(\cdot)}{ }_{u, L}^{p(\cdot)}}(\Phi)=\|\|\left\{f * \varphi_{j}\right\}_{j=0}^{\infty}\left\|_{l_{\nu}^{\alpha(\cdot), q(\cdot)}}\right\|_{\mathfrak{B}_{u, L}{ }^{p(\cdot)}}<\infty
$$

where $\varphi_{0} \in \mathcal{S}\left(\mathbb{R}^{n}\right)$ and $\varphi_{j}(x)=2^{j n} \varphi\left(2^{j} x\right), j \geq 1, \varphi \in \mathcal{S}\left(\mathbb{R}^{n}\right)$. The pair $\Phi=\left(\varphi_{0}, \varphi\right)$ satisfies

$$
\begin{aligned}
& \operatorname{supp}\left(\hat{\varphi}_{0}\right) \subset\left\{\xi \in \mathbb{R}^{n}:|\xi| \leq 1\right\}, \\
& \operatorname{supp}(\hat{\varphi}) \subset\left\{\xi \in \mathbb{R}^{n}: \frac{1}{2} \leq|\xi| \leq 2\right\},
\end{aligned}
$$

and $\hat{\varphi}_{0}+\hat{\varphi}_{1}=1$ on $\left\{\xi \in \mathbb{R}^{n}:|\xi| \leq 1\right\}$ and $\hat{\varphi}(2 \xi)+\hat{\varphi}(\xi)+\hat{\varphi}(\xi / 2)=1$ on $\left\{\xi \in \mathbb{R}^{n}\right.$ : $1 / 2 \leq|\xi| \leq 2\}$.

In $\left[9\right.$, Proposition 2.3], we find that if $u \equiv 1$ and $p(\cdot): \mathbb{R}^{n} \rightarrow(1, \infty)$ satisfies $p_{+}<$ $\infty$, then $\mathfrak{B}_{u, L^{p(\cdot)}}=L^{p(\cdot)}$. Thus, the Triebel-Lizorkin block space with variable exponent is an extension of the Triebel-Lizorkin space with variable exponent introduced in [17].

The family of function spaces defined in Definition 4.4 is new, even when $p(\cdot)=p, q(\cdot)=q$, and $\alpha(\cdot)=\alpha, 1<p, q<\infty,-\infty<\alpha<\infty$, are constant functions. In this case, we have the Triebel-Lizorkin block spaces. According to [9, Proposition 2.3], this is also a generalization of the classical Triebel-Lizorkin space (see [73]).

We establish a fundamental property of the Triebel-Lizorkin block spaces with variable exponents; namely, the function spaces given in Definition 4.4 are 
well defined. That is, it is independent of the functions $\Phi=\left(\varphi_{0}, \varphi\right)$ used in Definition 4.4. To prove this result, we recall the notion of $\phi-\psi$ transforms introduced in [22]-[24], and [25].

Let $\mathcal{Q}_{d}=\left\{Q_{\nu, k}: \nu \in \mathbb{N} \cup\{0\}, k \in \mathbb{Z}^{n}\right\}$ where $Q_{\nu, k}=\left\{\left(x_{1}, \ldots, x_{n}\right) \in \mathbb{R}^{n}: k_{j} \leq\right.$ $\left.2^{\nu} x_{j}<k_{j}+1, j=1, \ldots, n\right\}$ and $k=\left(k_{1}, \ldots, k_{n}\right)$. Denote the lower-left corner of the dyadic cube $Q$ by $x_{Q}=2^{-\nu} k$, denote the side length by $l(Q)$, and denote the Lebesgue measure of $Q$ by $|Q|$. Moreover, we write $\varphi_{Q}(x)=2^{n \nu} \varphi\left(2^{\nu} x-k\right)$ when $Q=Q_{\nu, k}$.

Let $\varphi_{0}, \psi_{0} \in \mathcal{S}\left(\mathbb{R}^{n}\right)$ and $\varphi, \psi \in \mathcal{S}\left(\mathbb{R}^{n}\right)$ satisfy

$$
\begin{aligned}
& \operatorname{supp} \hat{\varphi}_{0}, \operatorname{supp} \hat{\psi}_{0} \subseteq\left\{\xi \in \mathbb{R}^{n}:|\xi| \leq 2\right\}, \\
& \left|\hat{\varphi}_{0}(\xi)\right|,\left|\hat{\psi}_{0}(\xi)\right| \geq c>0 \quad \text { if }|\xi| \leq 5 / 3, \\
& \operatorname{supp} \hat{\varphi}, \operatorname{supp} \hat{\psi} \subseteq\left\{\xi \in \mathbb{R}^{n}: 1 / 2 \leq|\xi| \leq 2\right\} \text {, } \\
& |\hat{\varphi}(\xi)|,|\hat{\psi}(\xi)| \geq c>0 \quad \text { if } 3 / 5 \leq|\xi| \leq 5 / 3, \\
& \overline{\hat{\varphi}_{0}(\xi)} \hat{\psi}_{0}(\xi)+\sum_{\nu=1}^{\infty} \overline{\hat{\varphi}\left(2^{-\nu} \xi\right)} \hat{\psi}\left(2^{-\nu} \xi\right)=1, \quad \forall \xi \in \mathbb{R}^{n} .
\end{aligned}
$$

For any complex-valued sequence $s=\left\{s_{Q}\right\}_{Q \in \mathcal{Q}}$ and $f \in \mathcal{S}^{\prime}\left(\mathbb{R}^{n}\right)$, define $\mathrm{S}_{\varphi}(f)=$ $\left\{\left\langle f, \varphi_{Q}\right\rangle\right\}_{Q \in \mathcal{Q}}$ and $\mathrm{T}_{\psi}(s)=\sum_{Q \in \mathcal{Q}} s_{Q} \psi_{Q}$. We define the corresponding sequence space associated with $F_{q(\cdot), \mathfrak{B} u, L^{p(\cdot)}}^{\alpha(\cdot)}$.

\section{DEFINITION 4.5}

Let $(p, q, \alpha) \in \mathbb{S}$, and let $u$ be a Lebesgue measurable function satisfying (3.2) and

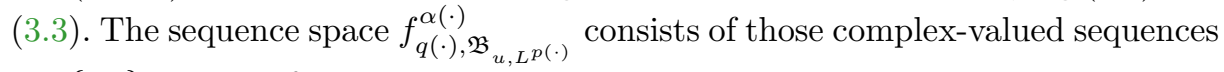
$s=\left\{s_{Q}\right\}_{Q \in \mathcal{Q}}$ satisfying

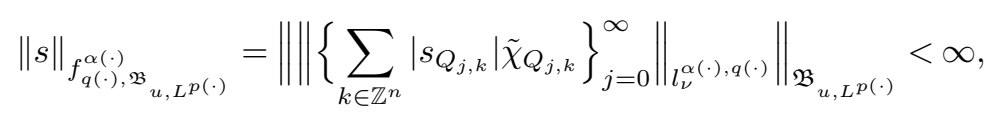

where $\tilde{\chi}_{Q}=|Q|^{-1 / 2} \chi_{Q}$ and $Q \in \mathcal{Q}_{d}$.

We next show the boundedness of the $\phi-\psi$ transforms and, hence, establish that $F_{q(\cdot), \mathfrak{B}_{u, L} p(\cdot)}^{\alpha(\cdot)}$ is well defined. Since the proof follows from the corresponding proofs of the Triebel-Lizorkin spaces with variable exponents (see [17]) and the TriebelLizorkin-Morrey spaces with variable exponents (see [35]), for brevity, we just outline the proof and refer the reader to [17, Theorem 3.4] and [35, Theorem 5.4] for details.

\section{THEOREM 4.2}

Let $(p, q, \alpha) \in \mathbb{S}$, and let $u$ be a Lebesgue measurable function satisfying (3.2) and (3.3). The $\varphi$-transform $S_{\varphi}: F_{q(\cdot), \mathfrak{B}_{u, L}(\cdot)}^{\alpha(\cdot)} \rightarrow f_{q(\cdot), \mathfrak{B}_{u, L^{p(\cdot)}}^{\alpha(\cdot)}}$ is bounded and the $\psi$ transform $T_{\psi}: f_{q(\cdot), \mathfrak{B}_{u, L} p(\cdot)}^{\alpha(\cdot)} \rightarrow F_{q(\cdot), \mathfrak{B}_{u, L} p(\cdot)}^{\alpha(\cdot)}$ is also bounded. Hence, $F_{q(\cdot), \mathfrak{B}_{u, L} p(\cdot)}^{\alpha(\cdot)}$ is well defined. 


\section{Proof}

For any $f \in F_{q(\cdot), \mathfrak{B}_{u, L} p(\cdot)}^{\alpha(\cdot)}$, we have

$$
\left\|S_{\varphi} f\right\|_{f_{q(\cdot), \mathfrak{B}_{u, L}}^{\alpha(\cdot)}}=\|\|\left\{\sum_{k \in \mathbb{Z}^{n}}\left|\left(\varphi_{\nu} * f\right)\left(x_{Q_{\nu, k}}\right)\right| \chi_{Q_{Q_{\nu, k}}}\right\}_{\nu=0}^{\infty}\|\|_{l_{\nu}^{\alpha(\cdot), q(\cdot)}} \|_{\mathfrak{B}_{u, L^{p(\cdot)}}} .
$$

Let $\eta_{m}(x)=(1+|x|)^{-m}$, and let $\eta_{\nu, m}=2^{n \nu} \eta_{m}\left(2^{\nu} x\right), \nu, m \in \mathbb{N} \cup\{0\}$. For sufficiently large $m$, Peetre's inequality (see [22, (2.11)], [73, Sections 1.4.1 and 1.4.2], or [17, Lemma A.6]) ensures that

$$
\left\|S_{\varphi} f\right\|_{F_{q(\cdot), B_{u, L}}^{\alpha(\cdot)}} \leq C\|\|\left\{\eta_{\nu, m} *\left|\varphi_{\nu} * f\right|\right\}_{\nu=0}^{\infty}\left\|_{l_{\nu}^{\alpha(\cdot), q(\cdot)}}\right\|_{\mathfrak{B}_{u, L} p(\cdot)} .
$$

Theorem 4.1 guarantees that

$$
\mathcal{T}\left(\left\{g_{\nu}\right\}_{\nu=0}^{\infty}\right)=\left\{\eta_{\nu, m} * g_{\nu}\right\}_{\nu=0}^{\infty}
$$

is a linear operator with singular kernel for $\left(l_{\nu}^{\alpha(\cdot), q(\cdot)}, l_{\nu}^{\alpha(\cdot), q(\cdot)}\right)$. Moreover, according to [17, Theorem 3.2], $\mathcal{T}$ is bounded on $L^{p(\cdot)}\left(l_{\nu}^{\alpha(\cdot), q(\cdot)}\right)$. Therefore, Theorem 3.3 gives that $\mathcal{T}$ is also bounded on $\mathfrak{B}_{u, L^{p(\cdot)}}\left(l_{\nu}^{\alpha(\cdot), q(\cdot)}\right)$. Consequently, the boundedness of $\mathcal{T}$ on $\mathfrak{B}_{u, L^{p(\cdot)}}\left(l_{\nu}^{\alpha(\cdot), q(\cdot)}\right)$ yields

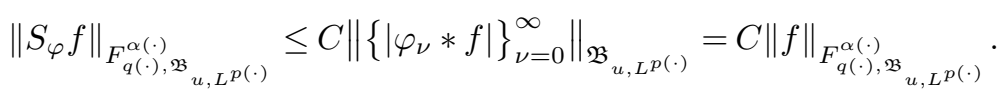

For the $\psi$-transform, let $s=\left\{s_{Q}\right\}_{Q \in \mathcal{Q}} \in f_{q(\cdot), \mathfrak{B}_{u, L} p(\cdot)}^{\alpha(\cdot)}$, and let $f=T_{\psi}(s)=$ $\sum_{Q \in \mathcal{Q}} s_{Q} \psi_{Q}$. Similar to the proof of [35, Lemma 5.3], we find that $f$ is well defined and belongs to $\mathcal{S}^{\prime}\left(\mathbb{R}^{n}\right)$. Since $\Phi=\left(\varphi_{0}, \varphi\right)$ satisfies the conditions in Definition 4.4 , the estimate from [24, p. 50] ensures that

$$
\left|\varphi_{\nu} * f\right| \leq C \sum_{\mu=\max (\nu-1,0)}^{\nu+1}\left(\left(\sum_{k \in \mathbb{Z}^{n}}\left|s_{Q_{\mu, k}}\right| \tilde{\chi}_{Q_{\mu, k}}\right) * \eta_{\mu, m}\right) .
$$

As $\mathcal{T}$ is bounded on $\mathfrak{B}_{u, L^{p(\cdot)}}\left(l_{\nu}^{\alpha(\cdot), q(\cdot)}\right)$ and the shift operators

$$
\begin{aligned}
& R\left(\left\{a_{j}\right\}_{j=0}^{\infty}\right)=\left\{a_{j+1}\right\}_{j=0}^{\infty}, \\
& L\left(\left\{a_{j}\right\}_{j=0}^{\infty}\right)=\left\{a_{j-1}\right\}_{j=0}^{\infty} \quad \text { with } a_{-1}=0
\end{aligned}
$$

are bounded uniformly on $l_{\nu}^{\alpha(\cdot), q(\cdot)}$, we have

$$
\begin{aligned}
& \left\|T_{\psi}(s)\right\|_{F_{q(\cdot), \mathfrak{B}}^{\alpha(\cdot)}{ }_{u, L^{p(\cdot)}}} \\
& =\|\|\left\{f * \varphi_{\nu}\right\}_{\nu=0}^{\infty} \|_{l_{\nu}^{\alpha(\cdot), q(\cdot)} \|_{\mathfrak{B}_{u, L^{p(\cdot)}}}}
\end{aligned}
$$

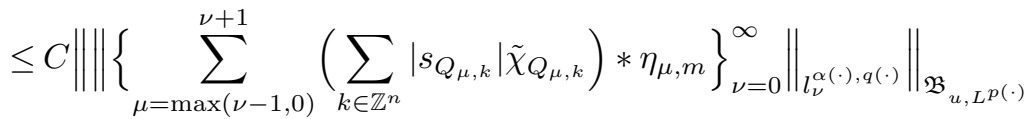

$$
\begin{aligned}
& \leq C\left\|\left\{\sum_{k \in \mathbb{Z}^{n}}\left|s_{Q_{\mu, k}}\right| \tilde{\chi}_{Q_{\mu, k}}\right\}_{\nu=0}^{\infty}\right\|\left\|_{F_{q(\cdot), \mathfrak{B}}^{\alpha(\cdot)}{ }_{u, L^{p(\cdot)}}}=C\right\| s \|_{f_{q(\cdot), \mathfrak{B}}^{\alpha(\cdot)}{ }_{u, L^{p(\cdot)}}}
\end{aligned}
$$

for some $C>0$. 
With the boundedness of the $\varphi-\psi$ transform, the rest of the proof follows from some simple modifications of the arguments from [24, Theorem 2.2]. For simplicity, we leave the details to the reader.

Furthermore, the Triebel-Lizorkin block spaces with variable exponents also possess atomic decompositions, molecular characterizations, and wavelet characterizations. Since the main purpose of this section is applying the operators with weakly singular kernel on function spaces, we skip the details of further studies of the Triebel-Lizorkin block spaces with variable exponents. The atomic decompositions and the molecular characterizations of Triebel-Lizorkin-Morrey spaces with variable exponents are given in [35, Theorems 6.11 and 6.12]. The reader is referred to [17] for the study of Triebel-Lizorkin spaces with variable exponents and [35] for the investigation of Triebel-Lizorkin-Morrey spaces with variable exponents.

Acknowledgment. The author would like to thank the reviewer for a careful reading of the paper and inspiration for further research.

\section{References}

[1] D. R. Adams and J. Xiao, Nonlinear potential analysis on Morrey spaces and their capacities, Indiana Univ. Math. J. 53 (2004), 1629-1663. MR 2106339. DOI 10.1512/iumj.2004.53.2470.

[2] - Morrey spaces in harmonic analysis, Ark. Mat. 50 (2012), 201-230. MR 2961318. DOI 10.1007/s11512-010-0134-0.

[3] A. Almeida, J. Hasanov, and S. Samko, Maximal and potential operators in variable exponent Morrey spaces, Georgian Math. J. 15 (2008), 195-208. MR 2428465.

[4] A. Almeida and P. Hästö, Besov spaces with variable smoothness and integrability, J. Funct. Anal. 258 (2010), 1628-1655. MR 2566313. DOI 10.1016/j.jfa.2009.09.012.

[5] K. F. Andersen and R. T. John, Weighted inequalities for vector-valued maximal functions and singular integrals, Studia Math. 69 (1980/81), 19-31. MR 0604351.

[6] C. Bennett and R. Sharpley, Interpolations of Operators, Pure Appl. Math. 129, Academic Press, Boston, 1988. MR 0928802.

[7] O. Blasco, A. Ruiz, and L. Vega, Non-interpolation in Morrey-Campanato and block spaces, Ann. Sc. Norm. Sup. Pisa Cl. Sci. (5) 28 (1999), 31-40.

MR 1679077.

[8] C. Capone, D. Cruz-Uribe, and A. Fiorenza, The fractional maximal operator and fractional integrals on variable $L^{p}$ spaces, Rev. Mat. Iberoam. 23 (2007), 743-770. MR 2414490. DOI 10.4171/RMI/511. 
[9] K. L. Cheung and K.-P. Ho, Boundedness of Hardy-Littlewood maximal operator on block spaces with variable exponent, Czechoslovak Math. J. 64(139) (2014), 159-171. MR 3247452. DOI 10.1007/s10587-014-0091-z.

[10] F. Chiarenza and M. Frasca, Morrey spaces and Hardy-Littlewood maximal function, Rend. Mat. Appl. (7) 7 (1987), 273-279. MR 0985999.

[11] D. Cruz-Uribe and A. Fiorenza, Variable Lebesgue Spaces: Foundation and Harmonic Analysis, Birkhäuser, Heidelberg, 2013. MR 3026953.

DOI 10.1007/978-3-0348-0548-3.

[12] D. Cruz-Uribe, A. Fiorenza, J. M. Martell, and C. Pérez, The boundedness of classical operators on variable $L^{p}$ spaces, Ann. Acad. Sci. Fenn. Math. 31 (2006), 239-264. MR 2210118.

[13] D. Cruz-Uribe, A. Fiorenza, and C. J. Neugebauer, The maximal function on variable $L^{p}$ spaces, Ann. Acad. Sci. Fenn. Math. 28 (2003), 223-238.

MR 1976842.

[14] D. Cruz-Uribe, J. M. Martell, and C. Pérez, Weights, Extrapolation and the Theory of Rubio de Francia, Oper. Theory Adv. Appl. 215, Birkhäuser, Basel, 2011. MR 2797562. DOI 10.1007/978-3-0348-0072-3.

[15] G. P. Curbera, J. García-Cuerva, J. M. Martell, and C. Pérez, Extrapolation with weights, rearrangement-invariant function spaces, modular inequalities and applications to singular integrals, Adv. Math. 203 (2006), 256-318.

MR 2231047. DOI 10.1016/j.aim.2005.04.009.

[16] L. Diening, P. Harjulehto, P. Hästö, and M. Růžička, Lebesgue and Sobolev Spaces with Variable Exponents, Lecture Notes in Math. 2017, Springer, Heidelberg, 2011. MR 2790542. DOI 10.1007/978-3-642-18363-8.

[17] L. Diening, P. Hästö, and S. Roudenko, Function spaces of variable smoothness and integrability, J. Funct. Anal. 256 (2009), 1731-1768. MR 2498558. DOI 10.1016/j.jfa.2009.01.017.

[18] L. Diening and M. Růžička, Calderón-Zygmund operators on generalized Lebesgue spaces $L^{p(\cdot)}$ and problems related to fluid dynamics, J. Reine Angew. Math. 563 (2003), 197-220. MR 2009242. DOI 10.1515/crll.2003.081.

[19] J. Diestel and J. J. Uhl, Jr., Vector Measures, Math. Surveys Mongr. 15, Amer. Math. Soc., Providence, 1977. MR 0453964.

[20] D. E. Edmunds and W. D. Evans, Hardy Operators, Function Spaces and Embeddings, Springer, Berlin, 2004. MR 2091115.

DOI 10.1007/978-3-662-07731-3.

[21] Eridani, H. Gunawan, E. Nakai, and Y. Sawano, Characterizations for the generalized fractional integral operators on Morrey spaces, Math. Inequal. Appl. 17 (2014), 761-777. MR 3235045.

[22] M. Frazier and B. Jawerth, Decomposition of Besov spaces, Indiana Univ. Math. J. 34 (1985), 777-799. MR 0808825. DOI 10.1512/iumj.1985.34.34041.

[23] "The $\phi$-transform and applications to distribution spaces" in Function Space and Applications (Lund, 1986), Lecture Notes in Math. 1302, Springer, Berlin, 1988, 223-246. MR 0942271. DOI 10.1007/BFb0078877. 
[24] A discrete transform and decompositions of distribution spaces, J. Funct. Anal. 93 (1990), 34-170. MR 1070037.

DOI 10.1016/0022-1236(90)90137-A.

[25] M. Frazier, B. Jawerth, and G. Weiss, Littlewood-Paley Theory and the Study of Function Spaces, CBMS Reg. Conf. Ser. Math. 79, Amer. Math. Soc., Providence, 1991. MR 1107300.

[26] J. García-Cuerva and J. L. Rubio de Francia, Weighted Norm Inequalities and Related Topics, North-Holland Math. Stud. 116, North-Holland, Amsterdam, 1985. MR 0807149.

[27] A. Gogatishvili and R. C. Mustafayev, New pre-dual space of Morrey space, J. Math. Anal. Appl. 397 (2013), 678-692. MR 2979604. DOI 10.1016/j.jmaa.2012.08.025.

[28] V. S. Guliyev, J. J. Hasanov, and S. G. Samko, Boundedness of the maximal, potential and singular operators in the generalized variable exponent Morrey spaces, Math. Scand. 107 (2010), 285-304. MR 2735097.

[29] V. S. Guliyev and S. G. Samko, Maximal, potential, and singular operators in the generalized variable exponent Morrey spaces on unbounded sets, J. Math. Sci. (N. Y.) 193 (2013), 228-248. MR 3077183. DOI 10.1007/s10958-013-1449-8.

[30] P. A. Hästö, Local-to-global results in variable exponent spaces, Math. Res. Lett. 16 (2009), 263-278. MR 2496743. DOI 10.4310/MRL.2009.v16.n2.a5.

[31] L. I. Hedberg and Y. Netrusov, An axiomatic approach to function spaces, spectral synthesis, and Luzin approximation, Mem. Amer. Math. Soc. 188, Amer. Math. Soc., Providence, 2007. MR 2326315. DOI 10.1090/memo/0882.

[32] K.-P. Ho, Remarks on Littlewood-Paley analysis, Canad. J. Math. 60 (2008), 1283-1305. MR 2462448. DOI 10.4153/CJM-2008-055-x.

[33] L Littlewood-Paley spaces, Math. Scand. 108 (2011), 77-102. MR 2780808.

[34] - Atomic decomposition of Hardy spaces and characterization of BMO via Banach function spaces, Anal. Math. 38 (2012), 173-185. MR 2958353. DOI 10.1007/s10476-012-0302-5.

[35] - Vector-valued singular integral operators on Morrey type spaces and variable Triebel-Lizorkin-Morrey spaces, Ann. Acad. Sci. Fenn. Math. 37 (2012), 375-406. MR 2987074. DOI 10.5186/aasfm.2012.3746.

[36] Atomic decompositions of weighted Hardy-Morrey spaces, Hokkaido Math. J. 42 (2013), 131-157. MR 3076303. DOI 10.14492/hokmj/1362406643.

[37] , The fractional integral operators on Morrey spaces with variable exponent on unbounded domains, Math. Inequal. Appl. 16 (2013), 363-373. MR 3059976. DOI 10.7153/mia-16-27.

[38] - Vector-valued maximal inequalities on weighted Orlicz-Morrey spaces, Tokyo J. Math. 36 (2013), 499-512. MR 3161571.

DOI 10.3836/tjm/1391177984. 
[39] - Atomic decomposition of Hardy-Morrey spaces with variable exponents, Ann. Acad. Sci. Fenn. Math. 40 (2015), 31-62. MR 3310072.

DOI 10.5186/aasfm.2015.4002.

[40] M. Izuki, Y. Sawano, and Y. Tsutsui, Variable Lebesgue norm estimates for BMO functions, II, Anal. Math. 40 (2014), 215-230. MR 3240224.

DOI 10.1007/s10476-014-0304-6.

[41] H. Jia and H. Wang, Decomposition of Hardy-Morrey spaces, J. Math. Anal. Appl. 354 (2009), 99-110. MR 2510421. DOI 10.1016/j.jmaa.2008.12.051.

[42] E. A. Kalita, Dual Morrey spaces (in Russian), Dokl. Akad. Nauk 361, no. 4 (1998), 447-449; English translation in Dokl. Math. 58 (1998), 85-87.

MR 1693091.

[43] V. Kokilashvili and A. Meskhi, Boundedness of maximal and singular operators in Morrey spaces with variable exponent, Armen. J. Math. 1 (2008), 18-28.

MR 2436241.

[44] Y. Liang, D. Yang, W. Yuan, Y. Sawano, and T. Ullrich, A new framework for generalized Besov-type and Triebel-Lizorkin-type spaces, Dissertationes Math. (Rozprawy Mat.) 489 (2013), 114 pp. MR 3099066. DOI 10.4064/dm489-0-1.

[45] J. Lindenstrauss and L. Tzafriri, Classical Banach Spaces, I and II, Springer, Berlin, 1996.

[46] Y. Meyer, M. H. Taibleson, and G. Weiss, Some functional analytic properties of the spaces $B_{q}$ generated by blocks, Indiana Univ. Math. J. 34 (1985), 493-515. MR 0794574. DOI 10.1512/iumj.1985.34.34028.

[47] C. B. Morrey, Jr., On the solutions of quasi-linear elliptic partial differential equations, Trans. Amer. Math. Soc. 43, no. 1 (1938), 126-166. MR 1501936. DOI 10.2307/1989904.

[48] E. Nakai, Hardy-Littlewood maximal operator, singular integral operators and the Riesz potentials on generalized Morrey spaces, Math. Nachr. 166 (1994), 95-103. MR 1273325. DOI 10.1002/mana.19941660108.

[49] , On generalized fractional integrals, Taiwanese J. Math. 5 (2001), 587-602. MR 1849780.

[50] , Orlicz-Morrey spaces and the Hardy-Littlewood maximal function, Studia Math. 188 (2008), 193-221. MR 2429821. DOI 10.4064/sm188-3-1.

[51] - Generalized fractional integrals on generalized Morrey spaces, Math. Nachr. 287 (2014), 339-351. MR 3163584. DOI 10.1002/mana.201200334.

[52] E. Nakai and Y. Sawano, Hardy spaces with variable exponents and generalized Campanato spaces, J. Funct. Anal. 262 (2012), 3665-3748. MR 2899976. DOI 10.1016/j.jfa.2012.01.004.

[53] H. Nakano, Modulared Semi-Ordered Linear Spaces, Maruzen, Tokyo, 1950. MR 0038565.

[54] Topology of Linear Topological Spaces, Maruzen, Tokyo, 1951. MR 0046560.

[55] A. Nekvinda, Hardy-Littlewood maximal operator on $L^{p(x)}(\mathbb{R})$, Math. Inequal. Appl. 7 (2004), 255-265. MR 2057644. DOI 10.7153/mia-07-28. 
[56] R. O'Neil, Fractional integration in Orlicz spaces, I, Trans. Amer. Math. Soc. 115 (1965), 300-328. MR 0194881.

[57] J. Peetre, On the theory of $\mathcal{L}_{p, \lambda}$ spaces, J. Funct. Anal. 4 (1969), 71-87. MR 0241965.

[58] J. L. Rubio de Francia, Factorization and extrapolation of weights, Bull. Amer. Math. Soc. (N.S.) 7 (1982), 393-395. MR 0663793. DOI 10.1090/S0273-0979-1982-15047-9.

[59] - "A new technique in the theory of $A_{p}$ weights" in Topics in Modern Harmonic Analysis, Vol. I, II (Turin/Milan, 1982), Ist. Naz. Alta Mat. Francesco Severi, Rome, 1983, 571-579. MR 0748875.

[60] Factorization theory and $A_{p}$ weights, Amer. J. Math. 106 (1984), 533-547. MR 0745140. DOI 10.2307/2374284.

[61] Y. Sawano, A note on Besov-Morrey spaces and Triebel-Lizorkin-Morrey spaces, Acta Math. Sin. (Engl. Ser.) 25 (2009), 1223-1242. MR 2524944. DOI 10.1007/s10114-009-8247-8.

[62] Y. Sawano, S. Sugano, and H. Tanaka, A note on generalized fractional integral operators on generalized Morrey spaces, Bound. Value Probl. 2009, no. 835865. MR 2587354.

[63] - Generalized fractional integral operators and fractional maximal operators in the framework of Morrey spaces, Trans. Amer. Math. Soc. 363, no. 12 (2011), 6481-6503. MR 2833565. DOI 10.1090/S0002-9947-2011-05294-3.

[64] _ Orlicz-Morrey spaces and fractional operators, Potential Anal. 36 (2012), 517-556. MR 2904632. DOI 10.1007/s11118-011-9239-8.

[65] Y. Sawano and H. Tanaka, Decompositions of Besov-Morrey spaces and Triebel-Lizorkin-Morrey spaces, Math. Z. 257 (2007), 871-905. MR 2342557. DOI 10.1007/s00209-007-0150-3.

[66] Y. Sawano and H. Wadade, On the Gagliardo-Nirenberg type inequality in the critical Sobolev-Morrey space, J. Fourier Anal. Appl. 19 (2013), 20-47. MR 3019769. DOI 10.1007/s00041-012-9223-8.

[67] Y. Sawano, D. Yang, and W. Yuan, New applications of Besov-type and Triebel-Lizorkin-type spaces, J. Math. Anal. Appl. 363 (2010), 73-85. MR 2559042. DOI 10.1016/j.jmaa.2009.08.002.

[68] F. Soria, Characterizations of classes of functions generated by blocks and associated Hardy spaces, Indiana Univ. Math. J. 34 (1985), 463-492. MR 0794573. DOI 10.1512/iumj.1985.34.34027.

[69] E. M. Stein, Harmonic Analysis: Real-Variable Methods, Orthogonality, and Oscillatory Integrals, Princeton Math. Ser. 43, Princeton Univ. Press, Princeton, 1993. MR 1232192.

[70] L. Tang and J. Xu, Some properties of Morrey type Besov-Triebel spaces, Math. Nachr. 278 (2005), 904-917. MR 2141966. DOI 10.1002/mana.200310281.

[71] A. Torchinsky, Interpolation of operations and Orlicz classes, Studia Math. 59 (1976/77), 177-207. MR 0438105. 
[72] R. Torres, Boundedness Results for Operators with Singular Kernels on Distribution Spaces, Mem. Amer. Math. Soc. 90, Amer. Math. Soc., Providence, 1991. MR 1048075.

[73] H. Triebel, Theory of Function Spaces, Monogr. Math. 78, Birkhäuser, Basel, 1983. MR 0781540. DOI 10.1007/978-3-0346-0416-1.

[74] D. Yang and W. Yuan, A new class of function spaces connecting Triebel-Lizorkin spaces and Q spaces, J. Funct. Anal. 255 (2008), 2760-2809. MR 2464191. DOI 10.1016/j.jfa.2008.09.005.

[75] _ New Besov-type spaces and Triebel-Lizorkin-type spaces including Q spaces, Math. Z. 265 (2010), 451-480. MR 2609320.

DOI 10.1007/s00209-009-0524-9.

[76] W. Yuan, W. Sickel, and D. Yang, Morrey and Campanato Meet Besov, Lizorkin and Triebel, Lecture Notes in Math. 2005, Springer, Berlin, 2010. MR 2683024. DOI 10.1007/978-3-642-14606-0.

[77] C. T. Zorko, Morrey spaces, Proc. Amer. Math. Soc. 98 (1986), 586-592. MR 0861756. DOI 10.2307/2045731.

Department of Mathematics and Information Technology, Hong Kong Institute of Education, Hong Kong, China; vkpho@ied.edu.hk 